\title{
MOLGW 1: many-body perturbation theory software for atoms, molecules, and clusters
}

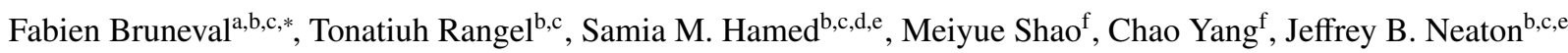 \\ ${ }^{a}$ CEA, DEN, Service de Recherches de Métallurgie Physique, F-91191 Gif-sur-Yvette, France \\ ${ }^{b}$ Molecular Foundry, Lawrence Berkeley National Laboratory, Berkeley, California 94720, United States \\ ${ }^{c}$ Department of Physics, University of California, Berkeley, California 94720, United States \\ ${ }^{d}$ Department of Chemistry, University of California, Berkeley, California 94720, United States \\ ${ }^{e}$ Kavli Energy Nanosciences Institute at Berkeley, Berkeley, California 94720, United States \\ ${ }^{f}$ Computational Research Division, Lawrence Berkeley National Laboratory, Berkeley, CA 94720, United States
}

\begin{abstract}
We summarize the MOLGW code that implements density-functional theory and many-body perturbation theory in a Gaussian basis set. The code is dedicated to the calculation of the many-body self-energy within the $G W$ approximation and the solution of the Bethe-Salpeter equation. These two types of calculations allow the user to evaluate physical quantities that can be compared to spectroscopic experiments. Quasiparticle energies, obtained through the calculation of the $G W$ self-energy, can be compared to photoemission or transport experiments, and neutral excitation energies and oscillator strengths, obtained via solution of the Bethe-Salpeter equation, are measurable by optical absorption. The implementation choices outlined here have aimed at the accuracy and robustness of calculated quantities with respect to measurments. Furthermore, the algorithms implemented in MOLGW allow users to consider molecules or clusters containing up to 100 atoms with rather accurate basis sets, and to choose whether or not to apply the resolution-of-the-identity approximation. Finally, we demonstrate the parallelization efficacy of the moLGW code over several hundreds of processors.
\end{abstract}

Keywords: Electronic structure of molecules; Many-body perturbation theory; $G W$ approximation; Bethe-Salpeter equation

\section{PROGRAM SUMMARY}

Program Title: MOLGW

Journal Reference:

Catalogue identifier:

Licensing provisions: GPL v3.0 [1]

Programming language: Fortran 2003 with a few C subrou-

tines, Python scripts

Number of processors used: 1-1024

Keywords: Electronic structure of molecules; Many-body perturbation theory; $G W$ approximation; Bethe-Salpeter equation

Classification:

External routines/libraries: LIBINT [2], LIBXC [3]

Nature of problem:

Prediction of the electronic structure of atoms, molecules, clusters with a particular interest in their spectroscopic features, such as quasiparticle energies and optical spectra. Solution method:

${ }^{*}$ Corresponding author.

E-mail address: fabien.bruneval@cea.fr

Preprint submitted to Computer Physics Communications
Using the $G W$ approximation to many-body perturbation theory, MOLGW calculates total-energies, quasiparticle energies, and optical excitations.

Running time:

From 30 seconds to a few hours

[1] http://www.gnu.org/copyleft/gpl.txt

[2] https://github.com/evaleev/libint

[3] http://www.tddft.org/programs/octopus/wiki/index.php/Libxc

\section{General presentation}

Over the years, many-body perturbation theory (MBPT) [1, 2, 3] has been demonstrated to lead to meaningful approximations for a variety of interesting electronic properties, but especially for those that involve excitations. Excited electronic states arise naturally when one connects calculations to, for example,

June 23, 2016 
experimental measurements of photoemission, inversephotoemission, charge transport, or optical absorption.

In the field of theoretical chemistry, the celebrated Möller-Plesset perturbation theory [4] is most commonly derived in increasing orders with respect to the bare Coulomb interaction $v$. However, for bulk metallic systems, the expansion with respect to $v$ gives rise to infinite terms, and is therefore inadequate. The use of the screened Coulomb interaction $W$, instead of the bare Coulomb interaction $v$, remedies this issue [2] and consequently, a comprehensive perturbative approach based on $W$ can be derived [5]. Its first-order approximation, namely the $G W$ approximation, has been used with great success for the last 30 years in extended systems to calculate the band gaps of solids $[6,7,8,9]$. In the past, the application of the $G W$ approximation to finite systems was rather infrequent $[10,11,12,13]$. But there has been much recent increase in interest $[14,15,16,17,18,19,20,21,22,23]$ in the application of the $G W$ approximation to problems involving atoms, molecules, and clusters, in part driven by the quest to develop efficient techniques to address mixed systems such as molecular junctions [24, 25, 26, 27, 28].

Several codes that implement MBPT in its $G W$ approximation for periodic systems are well-established and regularly utilized. To name a few of them, ABINIT [29], Yambo [30], SAX [31], vasp [32], and BerkeLEYGW [33] produce $G W$ quasiparticle energies and optical spectra from the Bethe-Salpeter equation (BSE). A few newer implementations have the advantage of a specific representation of $W$ to target large but still periodic systems [34, 35].

Implementations of the $G W$ approximation of MBPT for finite systems are much rarer, but their numbers have increased during the last few years and include RGWBS [13], FHI-AIMs [36], FIESTA [15], TURBOMOL [37], and MOLGW. While MOLGW has been employed in several previous studies [18, 23, 38, 39, 40], it has never been fully described in a single text.

In this article, we present version 1 of the MoLGW code. This software is particularly dedicated to the study of excited state properties of finite systems with MBPT using a Gaussian basis. MOLGW is a selfcontained code that is able to perform both the starting self-consistent field calculation in the generalized Kohn-Sham (gKS) framework and the MBPT posttreatment. The code can produce $G W$ quasiparticle energies and optical spectra through solution of the BSE.

The article is organized as follows: in Section 2, we quickly review the theoretical foundation of the $G W$ approximation and of the BSE; in Section 3, we detail the technical implementation; in Section 4, we describe the

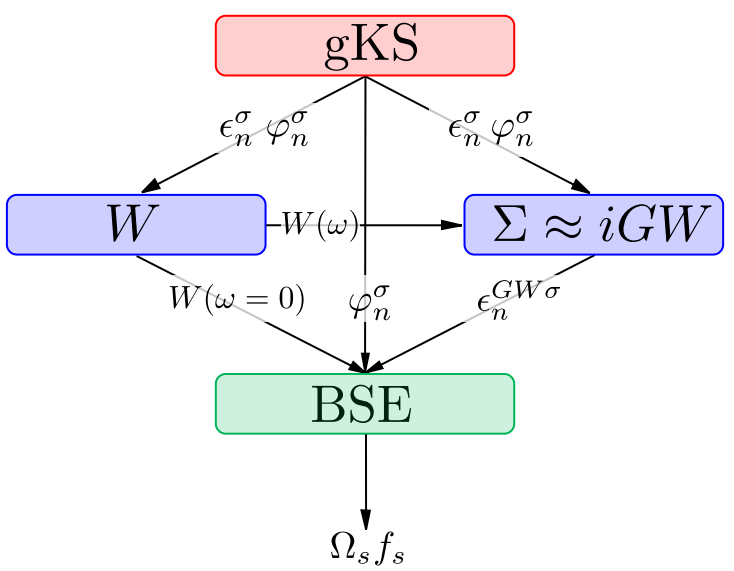

Figure 1: Typical workflow of perturbative $G W$ and BSE calculations.

code organization; in Section 5, we present a few validation studies; and in Section 6, we exhibit the parallelization capabilities of MOLGW.

In this article, Hartree atomic units will be used in all equations; wavefunctions will be considered real and consequently all conjugations will be dropped; state indexes $m$ and $n$ run over all states, empty and occupied; indexes $i$ and $j$ are limited to occupied states; and indexes $a$ and $b$ are limited to empty states.

\section{Theoretical background}

In most cases, an MBPT calculation is performed as a post-processing step based on a previous selfconsistent gKS run, as shown in Fig. 1. The optimal choice of gKS starting point, whether Hartree-Fock, a semi-local approximation, or a hybrid functional within density-functional theory (DFT), is not trivial for a given system and is currently an active area of research $[41,39,42,38,43,44]$. In any case, the wavefunctions and eigenvalues that result from the $\mathrm{gKS}$ calculation are used to produce the screened Coulomb interaction $W$ which is an ingredient in both the $G W$ approximation to the self-energy and in the BSE. The $G W$ self-energy is meant to produce accurate quasiparticle energies $\epsilon_{n}^{G W \sigma}$ that can be compared to photoemission and inversephotoemission spectroscopy, and these quasiparticle energies are in turn required input for the BSE solution. Finally, the solution to the BSE yields neutral excitation energies $\Omega_{s}$ and oscillator strengths $f_{s}$ that can be compared to optical absorption experiments.

Bearing in mind the different stages of the MBPT calculations, we are now prepared to introduce a few useful physical quantities. We do not intend to review all of 
the developments necessary for a complete derivation of MBPT. Rather, we sketch a rapid overview of the minimal knowledge required for the implementation of the $G W$ approximation to the self-energy and for the BSE solution. For a more complete understanding, we refer the reader to the review articles of Strinati [45], Aryasetiawan and Gunnarsson [8], and Onida et al. [46].

\subsection{GW self-energy}

The time-ordered Green's function is the central object in MBPT. It is a compact representation of additional electrons (for positive times) or missing electrons (for negative times) in the system.

In the specific case of electrons in a mean-field potential, which can be described with one-electron wavefunctions $\varphi_{m}^{\sigma}(\mathbf{r})$ and one-electron energies $\epsilon_{m}^{\sigma}$, the oneparticle Green's function reduces to

$$
G^{\sigma}\left(\mathbf{r}, \mathbf{r}^{\prime}, \omega\right)=\sum_{i} \frac{\varphi_{i}^{\sigma}(\mathbf{r}) \varphi_{i}^{\sigma}\left(\mathbf{r}^{\prime}\right)}{\omega-\epsilon_{i}^{\sigma}-\mathrm{i} \eta}+\sum_{a} \frac{\varphi_{a}^{\sigma}(\mathbf{r}) \varphi_{a}^{\sigma}\left(\mathbf{r}^{\prime}\right)}{\omega-\epsilon_{a}^{\sigma}+\mathrm{i} \eta}
$$

where the index $i$ runs over the occupied one-electron states, the index $a$ runs over the empty one-electron states, $\sigma$ is the spin channel, and $\eta$ is a small positive real number.

With this Green's function, one can build the noninteracting polarization $\chi_{0}$,

$\chi_{0}\left(\mathbf{r}, \mathbf{r}^{\prime}, \omega\right)=-\frac{\mathrm{i}}{2 \pi} \sum_{\sigma} \int d \omega_{1} G^{\sigma}\left(\mathbf{r}, \mathbf{r}^{\prime}, \omega+\omega_{1}\right) G^{\sigma}\left(\mathbf{r}^{\prime}, \mathbf{r}, \omega_{1}\right)$,

and the dielectric function $\varepsilon$ within the random-phase approximation (RPA)

$$
\varepsilon\left(\mathbf{r}, \mathbf{r}^{\prime}, \omega\right)=\delta\left(\mathbf{r}-\mathbf{r}^{\prime}\right)-\int d \mathbf{r}_{1} v\left(\mathbf{r}-\mathbf{r}_{1}\right) \chi_{0}\left(\mathbf{r}_{1}, \mathbf{r}^{\prime}, \omega\right) .
$$

The screened Coulomb interaction $W$ is then obtained as

$$
W\left(\mathbf{r}, \mathbf{r}^{\prime}, \omega\right)=\int d \mathbf{r}_{1} \varepsilon^{-1}\left(\mathbf{r}, \mathbf{r}_{1}, \omega\right) v\left(\mathbf{r}_{1}-\mathbf{r}^{\prime}\right)
$$

The $G W$ approximation is the first-order expansion of the self-energy in $W$. It reads

$$
\Sigma^{\sigma}\left(\mathbf{r}, \mathbf{r}^{\prime}, \omega\right)=\frac{\mathrm{i}}{2 \pi} \int d \omega_{1} e^{\mathrm{i} \eta \omega_{1}} G^{\sigma}\left(\mathbf{r}, \mathbf{r}^{\prime}, \omega+\omega_{1}\right) W\left(\mathbf{r}^{\prime}, \mathbf{r}, \omega_{1}\right),
$$

where the factor $e^{i \eta \omega_{1}}$ enforces the correct time-ordered form of the self-energy.

The $G W$ self-energy is most commonly evaluated within a perturbative approach, called $G_{0} W_{0}$ or alternatively "one-shot $G W$ ". In such a case, the $G W$ quasiparticle energies $\epsilon_{n}^{G W \sigma}$ are obtained through

$$
\epsilon_{n}^{G W \sigma}=\epsilon_{n}^{\sigma}+\left\langle\varphi_{n}^{\sigma}\left|\Sigma^{\sigma}\left(\epsilon_{n}^{G W \sigma}\right)-v_{x c}\right| \varphi_{n}^{\sigma}\right\rangle .
$$

Note that the self-energy is evaluated at the quasiparticle energy $\epsilon_{n}^{G W \sigma}$, therefore Eq. (6) must be solved numerically.

Some authors [42, 43, 47] advocate for iterating further the $G W$ equations by updating the eigenvalues in Eq. (1) to those from Eq. (6), while keeping the wavefunction fixed to the mean-field result. This procedure, usually called "evGW", appears as simple and robust.

Alternatively, Faleev et al. [48, 49, 50] proposed that the Hermitian part of a static approximation to the $G W$ self-energy be retained to produce new orthogonal wavefunctions that could be iterated as well. This approach is referred to as " $\mathrm{QS} G W$ " for quasiparticle selfconsistent $G W$

Nowadays, some groups [51, 14, 21] are exploring the possibility of fully self-consistent $G W$ calculations. As MOLGW does not have this capability, we will not mention this any further.

\subsection{Bethe-Salpeter equation}

The BSE is an equation for a contraction of the twoparticle Green's function $L$ that permits the calculation of polarizabilities that readily include the electron-hole interaction.

The BSE is usually assuming a static approximation of the $G W$ self-energy, which simplifies the equation into

$$
\begin{aligned}
& L^{\sigma \sigma^{\prime}}\left(\mathbf{r}_{1}, \mathbf{r}_{2} ; \mathbf{r}_{1}^{\prime}, \mathbf{r}_{2}^{\prime} ; \omega\right)= L_{0}^{\sigma \sigma^{\prime}}\left(\mathbf{r}_{1}, \mathbf{r}_{2} ; \mathbf{r}_{1}^{\prime}, \mathbf{r}_{2}^{\prime} ; \omega\right) \\
&+\int d \mathbf{r}_{3} d \mathbf{r}_{4} d \mathbf{r}_{5} d \mathbf{r}_{6} L_{0}^{\sigma \sigma^{\prime}}\left(\mathbf{r}_{1}, \mathbf{r}_{4} ; \mathbf{r}_{1}^{\prime}, \mathbf{r}_{3} ; \omega\right) \\
& \times\left[-\mathrm{i} v\left(\mathbf{r}_{3}-\mathbf{r}_{5}\right) \delta\left(\mathbf{r}_{3}-\mathbf{r}_{4}\right) \delta\left(\mathbf{r}_{5}-\mathbf{r}_{6}\right)\right. \\
&\left.+\mathrm{i} W\left(\mathbf{r}_{3}, \mathbf{r}_{4}, \omega=0\right) \delta\left(\mathbf{r}_{3}-\mathbf{r}_{5}\right) \delta\left(\mathbf{r}_{4}-\mathbf{r}_{6}\right) \delta_{\sigma \sigma^{\prime}}\right] \\
& \times L^{\sigma \sigma^{\prime}}\left(\mathbf{r}_{6}, \mathbf{r}_{2} ; \mathbf{r}_{5}, \mathbf{r}_{2}^{\prime} ; \omega\right),
\end{aligned}
$$

where $L_{0}$ stands for the non-interacting counterpart of $L$,

$$
\begin{aligned}
L_{0}^{\sigma \sigma^{\prime}}\left(\mathbf{r}_{1}, \mathbf{r}_{2} ; \mathbf{r}_{1}^{\prime}, \mathbf{r}_{2}^{\prime} ; \omega\right)=\frac{1}{2 \pi} \int & d \omega_{1} G^{\sigma}\left(\mathbf{r}_{1}, \mathbf{r}_{2}^{\prime}, \omega+\omega_{1}\right) \\
& \times G^{\sigma^{\prime}}\left(\mathbf{r}_{2}, \mathbf{r}_{1}^{\prime}, \omega_{1}\right)
\end{aligned}
$$

The electron-hole interaction arises from the statically screened Coulomb interaction $W\left(\mathbf{r}_{3}, \mathbf{r}_{4}, \omega=0\right)$, which is often named the BSE kernel $\Xi$.

A contraction of the indexes of $L_{0}$ gives back the usual non-interacting polarizability introduced in Eq. (2):

$$
\chi_{0}\left(\mathbf{r}_{1}, \mathbf{r}_{2}, \omega\right)=-\mathrm{i} \sum_{\sigma} L_{0}^{\sigma \sigma}\left(\mathbf{r}_{1}, \mathbf{r}_{2} ; \mathbf{r}_{1}, \mathbf{r}_{2} ; \omega\right)
$$


The fully interacting polarizability is obtained with the same index contraction:

$$
\chi\left(\mathbf{r}_{1}, \mathbf{r}_{2}, \omega\right)=-\mathrm{i} \sum_{\sigma} L^{\sigma \sigma}\left(\mathbf{r}_{1}, \mathbf{r}_{2} ; \mathbf{r}_{1}, \mathbf{r}_{2} ; \omega\right) .
$$

\section{Technical implementation}

In this section, we will describe how the general equations shown in the previous section translate when a finite basis set is introduced. The equations derived here closely match the notation and the forms used in the practical implementation of MOLGW and its source code.

\subsection{Gaussian basis sets}

In MOLGW, one-electron wavefunctions are expanded as linear combinations of atom-centered orbitals $\phi_{\mu}$ :

$$
\varphi_{n}^{\sigma}(\mathbf{r})=\sum_{\mu} C_{\mu n}^{\sigma} \phi_{\mu}(\mathbf{r}) .
$$

For numerical ease, the radial components of the atom-centered orbitals are chosen to be linear combinations of Gaussian orbitals or "contracted Gaussians" [3]. The angular component can be either "Cartesian"

$$
\phi_{\mu}(\mathbf{r})=x^{n_{x}} y^{n_{y}} z^{n_{z}} \sum_{b} c_{b} e^{-\alpha_{b} r^{2}}
$$

or "Pure"

$$
\phi_{\mu}(\mathbf{r})=\mathcal{Y}_{l m}(\hat{\mathbf{r}}) r^{l} \sum_{b} c_{b} e^{-\alpha_{b} r^{2}}
$$

where $\mathcal{Y}_{l m}(\hat{\mathbf{r}})$ are the real spherical harmonics. The choice of the angular part type or of the coefficients is dictated by the basis set selection. The transformations from Cartesian to pure are tabulated, for instance, in Ref. [52].

\subsection{Self-consistent field}

In practice, the $G W$ self-energy and the BSE solution require the knowledge of a mean-field starting point. With a gKS approach to obtain one-electron wavefunctions and energies, MOLGW solves the RoothanHall equation for spin restricted calculations and the Pople-Nesbet equations for those that are spin unrestricted [3]. This framework encompasses Hartree-Fock and semi-local approximations to DFT, as well as the hybrid functionals and range-separated hybrids.

The Roothan-Hall and Pople-Nesbet equations require a self-consistent solution to calculate the wavefunction coefficients $C^{\sigma}$ introduced in Eq. (11) and the

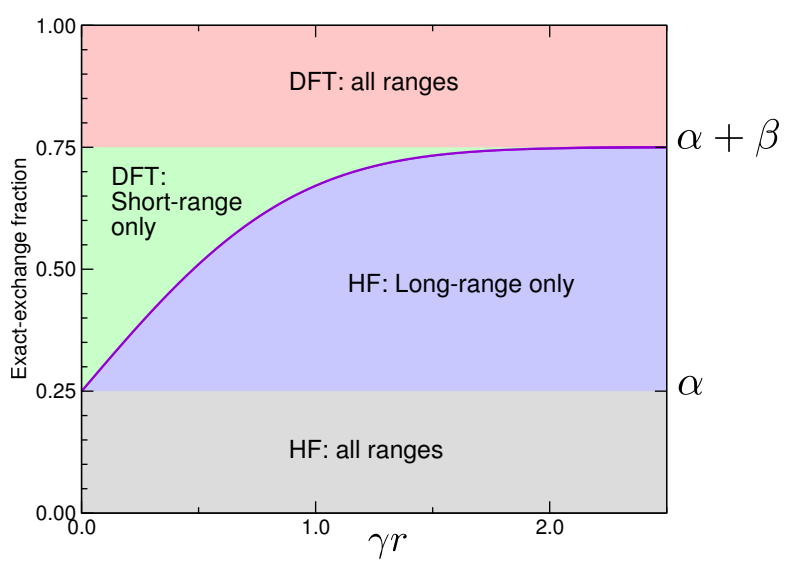

Figure 2: Exact-exchange fraction of a range-separated hybrid functional as a function of interelectron separation. The solid line represents the fraction of exact-exchange energy over the total (DFT and exact) exchange energy. The shaded areas show how the exchange is taken into account in the calculations at a given interelectron distance. Here we consider a range-separated hybrid functional with $\alpha=0.25$ and $\beta=0.50$ as an example.

one-electron energies $\epsilon^{\sigma}$ (which are subsequently stored in a diagonal matrix). These equations comprise the generalized diagonalization problem

$$
H^{\sigma} C^{\sigma}=S C^{\sigma} \epsilon^{\sigma}
$$

where $S$ is the basis function overlap matrix,

$$
S_{\mu v}=\int d \mathbf{r} \phi_{\mu}(\mathbf{r}) \phi_{\nu}(\mathbf{r})
$$

and $H^{\sigma}$ is the spin-dependent hamiltonian,

$$
\begin{aligned}
H_{\mu \nu}^{\sigma}=T_{\mu \nu}+V_{\text {ext } \mu \nu} & +J_{\mu \nu} \\
& -\alpha K_{\mu \nu}^{\sigma}-\beta K_{\mu \nu}^{\gamma \sigma}+\left(V_{x c}^{\sigma}\right)_{\mu \nu} .
\end{aligned}
$$

We show in Fig. 2 how the different components of the exchange are taken into account within the rangeseparated hybrid family of functionals, either calculated with the Fock exchange or with a DFT semi-local functional. The $\gamma$ parameter (an inverse length) governs the crossover between short and long range. The value of the $\alpha$ parameter is a measure of the amount of Fock exact-exchange. In Fig. 2, this fraction of the exchange is present at all ranges and it is graphically represented by the lower gray area. The $\beta$ parameter dictates the proportion of long-range Fock exchange. In Fig. 2, because of positive $\beta$, the amount of exact-exchange increases with interelectron distance. It is graphically represented by the central blue area. The DFT semi-local exchange contribution complements the Fock exchange 
so that a full account of the exchange is recovered at any interelectron separation. Therefore, the amount of DFT semi-local exchange decreases with interelectron distance for positive $\beta$. The DFT exchange contribution consists of two parts: a standard exchange part, which is constant for any distance and which is represented by the upper red area in Fig. 2; and a shortrange part, which precisely complements the long-range exact-exchange Fock contribution. This latter contribution is proportional to $\beta$ and is represented by the central green area in Fig. 2. Note that the standard DFT exchange is introduced proportional to $1-\alpha-\beta$ so that the exchange is fully accounted for irrespective of range. Finally, it should be stressed that the DFT exchangecorrelation potential $V_{x c}$ is a function of $\alpha, \beta$, and $\gamma$, even though it is not explicitly noted.

Functionals with no range separation, such as B3LYP [53] or PBE0 [54], can be obtained by setting $\beta$ or $\gamma$ to 0 . The functionals with the correct asymptotics [55] enforce $\alpha+\beta=1$. The short-range only functionals, such as HSE06 [56, 57], use $\beta=-\alpha$. With this framework, we have then the full flexibility to account for most current hybrid functionals on the market.

Let us detail now the terms in the Hamiltonian in Eq. (16). It consists of a kinetic energy term

$$
T_{\mu \nu}=-\frac{1}{2} \int d \mathbf{r} \lim _{\mathbf{r}^{\prime} \rightarrow \mathbf{r}} \phi_{\mu}(\mathbf{r}) \nabla_{\mathbf{r}^{\prime}}^{2} \phi_{\nu}\left(\mathbf{r}^{\prime}\right),
$$

an external potential energy term

$$
V_{\text {ext } \mu \nu}=-\sum_{a} \int d \mathbf{r} \phi_{\mu}(\mathbf{r}) \frac{Z_{a}}{\left|\mathbf{r}-\mathbf{R}_{a}\right|} \phi_{\nu}(\mathbf{r}),
$$

where $Z_{a}$ and $\mathbf{R}_{a}$ are the charge and the position of the nuclei, and electron-electron interaction terms.

First, the Hartree term accounts for the classical electron-electron repulsion. It reads

$$
J_{\mu \nu}=\sum_{\lambda \tau}(\mu \nu \mid \lambda \tau) \sum_{\sigma} P_{\lambda \tau}^{\sigma},
$$

where the density matrix $P$ is introduced as

$$
P_{\mu \nu}^{\sigma}=\sum_{n} f_{n}^{\sigma} C_{\mu n}^{\sigma} C_{v n}^{\sigma}
$$

with $f_{n}^{\sigma}$ the occupation number, as well as the electron repulsion integrals in Mulliken notation:

$$
(\mu \nu \mid \lambda \tau)=\iint d \mathbf{r} d \mathbf{r}^{\prime} \phi_{\mu}(\mathbf{r}) \phi_{\nu}(\mathbf{r}) \frac{1}{\left|\mathbf{r}-\mathbf{r}^{\prime}\right|} \phi_{\lambda}\left(\mathbf{r}^{\prime}\right) \phi_{\tau}\left(\mathbf{r}^{\prime}\right) .
$$

In order to account for hybrid functionals (and rangeseparated hybrids), we have explicitly written the allrange exchange matrix, rescaled with a factor $\alpha$ in Eq. (16),

$$
K_{\mu \nu}^{\sigma}=\sum_{\lambda \tau} P_{\lambda \tau}^{\sigma}(\mu \lambda \mid \tau v)
$$

and the long-range only exchange matrix, rescaled with a factor $\beta$ in Eq. (16),

$$
K_{\mu \nu}^{\gamma \sigma}=\sum_{\lambda \tau} P_{\lambda \tau}^{\sigma}(\mu \lambda \mid \tau v)^{\gamma},
$$

where the long-range electron repulsion integrals are defined as

$(\mu \nu \mid \lambda \tau)^{\gamma}=\iint d \mathbf{r} d \mathbf{r}^{\prime} \phi_{\mu}(\mathbf{r}) \phi_{\nu}(\mathbf{r}) \frac{\operatorname{erf}\left(\gamma\left|\mathbf{r}-\mathbf{r}^{\prime}\right|\right)}{\left|\mathbf{r}-\mathbf{r}^{\prime}\right|} \phi_{\lambda}\left(\mathbf{r}^{\prime}\right) \phi_{\tau}\left(\mathbf{r}^{\prime}\right)$.

In practice, the electron repulsion integrals are evaluated using recursion formulas [58] as implemented in the LIBINT library [59]. The range-separated integrals are obtained simply by changing the initial term of the recursion [56].

A priori integral screening with the Schwarz inequality

$$
(\mu \nu \mid \lambda \tau) \leq \sqrt{(\mu \nu \mid \mu \nu)} \sqrt{(\lambda \tau \mid \lambda \tau)}
$$

can drastically reduce the number of 4-center integrals to be calculated [60], and we make use of this here.

The density-functional exchange-correlation potential $V_{x c}$ is then evaluated with the expression [61]

$$
\begin{aligned}
&\left(V_{x c}^{\uparrow}\right)_{\mu v}=\left\langle\mu\left|v_{x c}\left[\rho^{\sigma}(\mathbf{r}), \nabla_{\mathbf{r}} \rho^{\sigma}(\mathbf{r})\right]\right| v\right\rangle \\
&= \int d \mathbf{r}\left\{\frac{\partial \epsilon_{x c}}{\partial \rho^{\uparrow}} \phi_{\mu}(\mathbf{r}) \phi_{v}(\mathbf{r})\right. \\
&+ {\left[2 \frac{\partial \epsilon_{x c}}{\partial \gamma_{\uparrow \uparrow}} \nabla_{\mathbf{r}} \rho^{\uparrow}(\mathbf{r})+\frac{\partial \epsilon_{x c}}{\partial \gamma_{\uparrow \downarrow}} \nabla_{\mathbf{r}} \rho^{\downarrow}(\mathbf{r})\right] } \\
&\left.\cdot \nabla_{\mathbf{r}}\left[\phi_{\mu}(\mathbf{r}) \phi_{v}(\mathbf{r})\right]\right\},
\end{aligned}
$$

where $\epsilon_{x c}$ is the exchange-correlation energy density (per unit volume), and where

$$
\gamma_{\sigma \sigma^{\prime}}=\nabla_{\mathbf{r}} \rho^{\sigma}(\mathbf{r}) \cdot \nabla_{\mathbf{r}} \rho^{\sigma^{\prime}}(\mathbf{r}) .
$$

The corresponding expression for $V_{x c}^{\downarrow}$ is analogous. In MOLGW, the values of $\epsilon_{x c}$ and its derivatives are obtained from the external library LIBXC [62] .

The density and the density gradient are obtained from the density matrix as

$$
\begin{aligned}
\rho^{\sigma}(\mathbf{r}) & =\sum_{\mu \nu} P_{\mu \nu}^{\sigma} \phi_{\mu}(\mathbf{r}) \phi_{\nu}(\mathbf{r}) \\
\nabla \rho^{\sigma}(\mathbf{r}) & =\sum_{\mu \nu} P_{\mu \nu}^{\sigma} \nabla_{\mathbf{r}}\left[\phi_{\mu}(\mathbf{r}) \phi_{\nu}(\mathbf{r})\right] .
\end{aligned}
$$


As is standard in quantum chemistry, the integration in Eq. (27) for the evaluation of the matrix elements $\left(V_{x c}^{\sigma}\right)_{\mu \nu}$ is performed by numerical quadrature. First, the space is partitioned in smooth regions around each atom using Becke [63] or SSF schemes [64]. Then, each atom carries its own quadrature points in spherical coordinates combining an angular Lebedev-Laikov mesh [65] and a radial mesh [66].

Finally, the Roothan-Hall or Pople-Nesbet equations should be solved self-consistently because of the dependence of the terms $J, K, K^{\gamma}, V_{x c}$ on the density matrix. The method due to Pulay, extremely efficient $[67,68]$ and rather robust, is used here.

\subsection{Auxiliary basis set}

In order to reduce the computational and the memory costs associated with the 4-center integrals $(\mu \nu \mid \lambda \tau)$, the introduction of an auxiliary basis set to represent the orbital products $\phi_{\mu}(\mathbf{r}) \phi_{\nu}(\mathbf{r})$ has been proposed [69, 70, 71]. This method, known as the resolution-of-the-identity method, allows one to approximate the 4-center integrals in terms of 2- and 3-center integrals, as

$$
(\mu \nu \mid \lambda \tau) \approx \sum_{P Q}(\mu \nu \mid P)(P \mid Q)^{-1}(Q \mid \lambda \tau)
$$

where the 3-center integrals read

$$
(\mu \nu \mid P)=\int d \mathbf{r} d \mathbf{r}^{\prime} \phi_{\mu}(\mathbf{r}) \phi_{v}(\mathbf{r}) \frac{1}{\left|\mathbf{r}-\mathbf{r}^{\prime}\right|} \phi_{P}\left(\mathbf{r}^{\prime}\right)
$$

and the 2-center integrals are

$$
(P \mid Q)=\int d \mathbf{r} d \mathbf{r}^{\prime} \phi_{P}(\mathbf{r}) \frac{1}{\left|\mathbf{r}-\mathbf{r}^{\prime}\right|} \phi_{Q}\left(\mathbf{r}^{\prime}\right) .
$$

The index running over the auxiliary basis are labeled with capital letters $P, Q$, etc.

The quality of the approximation in Eq. (31) depends on the quality of the auxiliary basis set. The basis sets proposed by Weigend [71] permits an accurate expansion of the orbital products that are used in both the Hartree term $J$ and in the Fock exchange term $K$. This method is called "RI-JK".

In practice, the 2-center integral matrix $(P \mid Q)$ is positive definite and its square-root can be calculated so as to transform Eq. (31) into

$$
(\mu \nu \mid \lambda \tau) \approx \sum_{P} M_{\mu \nu}^{P} M_{\lambda \tau}^{P}
$$

where

$$
M_{\mu \nu}^{P}=\sum_{Q}(\mu \nu \mid Q)(Q \mid P)^{-1 / 2}
$$

This is computationally simpler to evaluate ( $N$ operations instead of $N^{2}$ ).

In MOLGW, one can choose whether or not to evaluate the Hartree term $J$ and the Fock term $K$ with the resolution-of-the-identity (RI) approximation.

\subsection{Polarizability in the state product basis}

Both the $G W$ calculations and the BSE require the calculation of the polarizability. In standard $G W$ calculations, it is obtained within the random phase approximation (RPA), whereas in the BSE, one needs to introduce the additional kernel $\Xi$ described in Section 2.2. In MOLGW, whatever the approximation, the calculation of the polarizability follows the same procedure. The advantage of this is that the spectral decomposition of the polarizability may be obtained so that its evaluation at any frequency is exact and, as a consequence, the $G W$ self-energy convolution can be calculated analytically.

The BSE equation in Eq. (7) can be written in terms of a product basis of occupied to unoccupied states (and the converse). This transformation is precisely the same one frequently used to represent timedependent Hartree-Fock (TD-HF) and time-dependent density-functional theory (TD-DFT) within linear response $[72,73,74]$. Due to the symmetry between resonant (occupied to empty) and anti-resonant (empty to occupied) transitions, these equations have a very specific block form:

$$
\left(\begin{array}{rr}
A & B \\
-B & -A
\end{array}\right)\left(\begin{array}{c}
X^{s} \\
Y^{s}
\end{array}\right)=\left(\begin{array}{c}
X^{s} \\
Y^{s}
\end{array}\right) \Omega_{s},
$$

where $\Omega_{s}$ are the neutral excitation energies and $\left(X^{s}, Y^{s}\right)$ are the corresponding eigenvectors.

The only difference between RPA, TD-HF, TD-DFT and BSE lies in the specific expression of the matrix elements in $A$ and $B$. Let $i$ and $j$ indexes stand for occupied states and $a$ and $b$ for unoccupied states. The expressions for these elements read

$$
\begin{aligned}
A_{i a \sigma}^{j b \sigma^{\prime}}= & \left(\epsilon_{a}^{\sigma}-\epsilon_{i}^{\sigma}\right) \delta_{i j} \delta_{a b} \delta_{\sigma \sigma^{\prime}} \\
& -\left(i a \sigma \mid j b \sigma^{\prime}\right)+\Xi_{i a \sigma}^{j b \sigma^{\prime}} \\
B_{i a \sigma}^{j b \sigma^{\prime}=} & -\left(i a \sigma \mid b j \sigma^{\prime}\right)+\Xi_{i a \sigma}^{b j \sigma^{\prime}},
\end{aligned}
$$

where the operator $\Xi$ stands for the kernel of the selected approximation. In the specific case of RPA, $\Xi=0$. The expression of $\Xi$ in BSE will be addressed further below.

The molecular orbital electron repulsion integrals are defined as

$\left(i a \sigma \mid j b \sigma^{\prime}\right)=\iint d \mathbf{r} d \mathbf{r}^{\prime} \varphi_{i}^{\sigma}(\mathbf{r}) \varphi_{a}^{\sigma}(\mathbf{r}) \frac{1}{\left|\mathbf{r}-\mathbf{r}^{\prime}\right|} \varphi_{j}^{\sigma^{\prime}}\left(\mathbf{r}^{\prime}\right) \varphi_{b}^{\sigma^{\prime}}\left(\mathbf{r}^{\prime}\right)$ 
and are obtained by the canonical transform

$$
\left(i a \sigma \mid j b \sigma^{\prime}\right)=\sum_{\mu \nu \lambda \tau} C_{\mu i}^{\sigma} C_{v a}^{\sigma} C_{\lambda j}^{\sigma^{\prime}} C_{\tau b}^{\sigma^{\prime}}(\mu \nu \mid \lambda \tau)
$$

When the resolution-of-the-identity approximation is used, the scaling of the transform above can be improved:

$$
\begin{aligned}
\left(i a \sigma \mid j b \sigma^{\prime}\right) \approx \sum_{P}\left(\sum_{\mu \nu} C_{\mu i}^{\sigma} C_{\nu a}^{\sigma} M_{\mu \nu}^{P}\right) & \\
& \times\left(\sum_{\lambda \tau} C_{\lambda j}^{\sigma^{\prime}} C_{\tau b}^{\sigma^{\prime}} M_{\lambda \tau}^{P}\right) .
\end{aligned}
$$

Due to the peculiar block structure of Eq. (36), which induces many symmetries, it can be recast into a smaller matrix diagonalization, after some algebra [73]:

$$
C Z^{s}=Z^{s} \Omega_{s}^{2}
$$

where $C=(A-B)^{1 / 2}(A+B)(A-B)^{1 / 2}$ is a symmetric matrix that is half the size of the initial problem in Eq. (36). The above expression assumes matrix $(A-B)$ to be positive definite. From the knowledge of an eigenvector $Z^{s}$, one can recover both $X^{s}$ and $Y^{s}$ as

$$
\begin{aligned}
X^{s} & =\frac{1}{2}\left[\Omega_{s}^{-1 / 2}(A-B)^{1 / 2}+\Omega_{s}^{1 / 2}(A-B)^{-1 / 2}\right] Z^{s} \\
Y^{s} & =\frac{1}{2}\left[\Omega_{s}^{-1 / 2}(A-B)^{1 / 2}-\Omega_{s}^{1 / 2}(A-B)^{-1 / 2}\right] Z^{s} .
\end{aligned}
$$

The equations for $X^{s}$ and $Y^{s}$ imply normalization conditions that can be recast into a matrix form:

$$
\left(\begin{array}{rr}
X & -Y \\
-Y & X
\end{array}\right)^{T}\left(\begin{array}{ll}
X & Y \\
Y & X
\end{array}\right)=I,
$$

where the left-hand matrix contains the left eigenvectors and the right-hand matrix contains the right eigenvectors of Eq. (36). A more efficient way of solving Eq. (36) that avoids the computation of the matrix square-root will be discussed in Section 6.2.

Once the eigenvalues $\Omega_{s}$ and the eigenvectors $\left(X^{s}, Y^{s}\right)$ have been obtained through the diagonalization of Eq. (41) and the matrix multiplications in Eqs. (42a$42 \mathrm{~b}$ ), the spectral representation of the polarizability follows thanks to Eq. (10). Then, using the definition of the screened Coulomb interaction in Eq. (4), $W$ reads

$$
\begin{aligned}
W_{m n \sigma}^{o p \sigma^{\prime}}(\omega)=( & \left(m n \sigma \mid o p \sigma^{\prime}\right)+\sum_{s} w_{m n \sigma}^{s} w_{o p \sigma^{\prime}}^{s} \\
& \times\left(\frac{1}{\omega-\Omega_{s}+\mathrm{i} \eta}-\frac{1}{\omega+\Omega_{s}-\mathrm{i} \eta}\right),
\end{aligned}
$$

where the residues $w_{m n \sigma}^{s}$ are defined as

$$
w_{m n \sigma}^{s}=\sum_{i a \sigma^{\prime}}\left(m n \sigma \mid i a \sigma^{\prime}\right)\left(X_{i a \sigma^{\prime}}^{s}+Y_{i a \sigma^{\prime}}^{s}\right) .
$$

Note that once again the resonant/antiresonant symmetry has been used in Eq. (44) to produce a more compact expression.

\subsection{Many-body total energies}

The $G W$ approximation has been mentioned so far with the aim of obtaining self- and quasiparticle energies, but it can also be used to calculate total energies $[5,75,12,21]$.

There are several expressions for the $G W$ total energy that all match for a self-consistent solution of the Green's function $G$. However, they differ when evaluated with a perturbative $G$ obtained from a gKS starting point $[12,76]$. One of these expressions, the Klein functional [77], is particularly interesting, since it yields a stationary expression for the total energy and it matches the RPA total energy obtained in the DFT framework [78, 79, 80].

In practice, when calculating the Klein functional from a gKS potential, Furche [81] proposed a very compact formula that avoids the numerical integration over the coupling constant and over frequencies. The expression only involves the results already calculated for the polarizability anyway:

$$
E_{c}^{\mathrm{RPA}}=-\frac{1}{2} \sum_{s} \Omega_{s}+\frac{1}{2} \sum_{i a \sigma} \epsilon_{a}^{\sigma}-\epsilon_{i}^{\sigma}-(i a \sigma \mid i a \sigma) .
$$

This last equation is nothing more than the difference between the eigenvalues and the diagonal terms of Eq. (36).

\subsection{GW self-energy}

With the spectral decomposition of $W$ at hand, and using the residue theorem, it is possible to give an analytical expression for the $G W$ self-energy convolution in Eq. (5).

The first term on the right-hand side of Eq. (44) gives the usual Fock exchange term. The rest yields the correlation part of the self-energy $\Sigma_{c}$, whose diagonal matrix elements read

$$
\begin{aligned}
\left\langle\varphi_{n}^{\sigma}\left|\Sigma_{c}^{\sigma}(\omega)\right| \varphi_{n}^{\sigma}\right\rangle=\sum_{i s} \frac{w_{n i \sigma}^{s} w_{n i \sigma}^{s}}{\omega-\epsilon_{i}^{\sigma}+\Omega_{s}-\mathrm{i} \eta} \\
+\sum_{a s} \frac{w_{n a \sigma}^{s} w_{n a \sigma}^{s}}{\omega-\epsilon_{a}^{\sigma}-\Omega_{s}+\mathrm{i} \eta}
\end{aligned}
$$



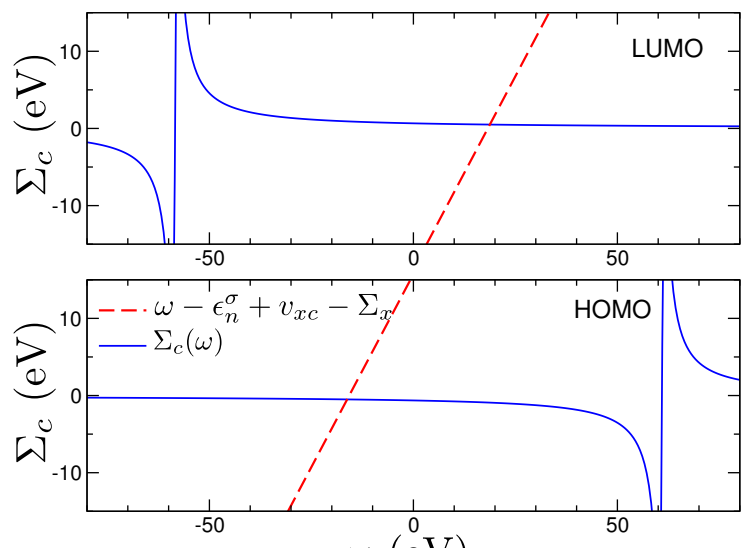

$\omega(\mathrm{eV})$

Figure 3: Graphical solution of the quasiparticle equation for at $\mathrm{H}_{2}$ molecule in the minimal basis (STO-3G) for the LUMO state (upper panel) and the HOMO state (lower panel). The quasiparticle energy is defined at the intersection between the correlation part of the selfenergy (solid blue line) and of the line $\omega-\epsilon_{n}^{\sigma}+v_{x c}-\Sigma_{x}$ (dashed red line).

where we use again the convention that index $a$ runs over empty states and $i$ runs over occupied states.

Hedin's static approximation to $G W$, named "Coulomb-hole plus screened exchange" (COHSEX) $[5,82]$, can be derived from Eq. (47) by considering the limit of $\Omega_{s} \gg\left|\omega-\epsilon_{m}^{\sigma}\right|$ :

$$
\left\langle\varphi_{n}^{\sigma}\left|\Sigma_{c}^{\sigma}\right| \varphi_{n}^{\sigma}\right\rangle=2 \sum_{i s} \frac{w_{n i \sigma}^{s} w_{n i \sigma}^{s}}{\Omega_{s}}-\sum_{m s} \frac{w_{n m \sigma}^{s} w_{n m \sigma}^{s}}{\Omega_{s}}
$$

The first term in Eq. (48) is the pure screened part of the screened exchange, whereas the second one is the Coulomb hole.

As an example, Fig. 3 shows the solution of the quasiparticle equation in Eq. (6) for the $\mathrm{H}_{2}$ molecule within $G W$ based on a Hartree-Fock starting point $\left(G_{0} W_{0} @ \mathrm{HF}\right)$ using the minimal STO-3G basis. In a minimal basis, $\mathrm{H}_{2}$ has two states: the highest-occupied molecular orbital (HOMO) and the lowest-unoccupied molecular orbital (LUMO). As a consequence, there is only one pair of neutral excitation energies at $\pm \Omega_{s}$ and hence, the $G W$ self-energy only shows two poles at $\epsilon_{\mathrm{HOMO}}-\Omega_{s}$ and at $\epsilon_{\mathrm{LUMO}}+\Omega_{s}$ as inferred from Eq. (47). When the poles of the self-energy are distant from the quasiparticle energies, a linearization of the self-energy can be carried out. MOLGW performs both the linearized and the graphical solution to the quasiparticle equation.

\subsection{BSE optical excitations}

The BSE is a particular case of the polarizability equation written in Eqs. (36, 37a, 37b). In the standard static BSE [83, 84, 85, 86, 46], the one-particle energies are the $G W$ quasiparticle energies and the kernel $\Xi$ reduces to

$$
\Xi_{i a \sigma}^{j b \sigma^{\prime}}=\delta_{\sigma \sigma^{\prime}} W_{i j \sigma}^{a b \sigma}(\omega=0),
$$

where $W(\omega=0)$ is the RPA screened Coulomb interaction evaluated at $\omega=0$.

Once the spectral decomposition of $W$ has been calculated for the $G W$ self-energy, it can be reused for the BSE kernel $\Xi$. In practice, $\Xi$ is obtained by introducing Eq. (44) into Eq. (49):

$$
\Xi_{i a \sigma}^{j b \sigma^{\prime}}=\delta_{\sigma \sigma^{\prime}}\left[(i j \sigma \mid a b \sigma)-2 \sum_{s} \frac{w_{i j \sigma}^{s} w_{a b \sigma}^{s}}{\Omega_{s}}\right] .
$$

Once the BSE is solved, we can connect the results of the calculations to experiment, either by directly comparing the neutral excitations energies $\Omega_{s}$ or by evaluating the optical spectra. For instance, the photoabsorption cross section tensor $\sigma_{x x^{\prime}}(\omega)$ can be calculated [73] as

$$
\begin{aligned}
\sigma_{x x^{\prime}}(\omega)=- & \frac{4 \pi \omega}{c} \sum_{s} f_{x}^{s} f_{x^{\prime}}^{s} \\
& \times\left(\frac{1}{\omega-\Omega_{s}+\mathrm{i} \eta}-\frac{1}{\omega+\Omega_{s}+\mathrm{i} \eta}\right),
\end{aligned}
$$

with the oscillator strength $f_{x}^{s}$ obtained as

$$
f_{x}^{s}=\sum_{i a \sigma}\langle i \sigma|\hat{x}| a \sigma\rangle\left(X_{i a \sigma}^{s}+Y_{i a \sigma}^{s}\right) .
$$

The symbol $\hat{x}$ is the position operator along the $x$ direction in space.

The oscillator strengths should add up to the number of electrons in the system $N_{e l}$ according to the ThomasReiche-Kuhne sum rule [73]

$$
\frac{2}{3} \sum_{s} \sum_{x}\left(f_{x}^{s}\right)^{2} \Omega_{s}=N_{e l} .
$$

However this relation is fulfilled only in the limit of a complete basis set.

Finally, we would like to mention that the so-called Tamm-Dancoff approximation can be used within BSE. This approximation consists in setting the $B$ block to zero in Eq. (36). The BSE for a spin-triplet final state can be evaluated as shown, for instance in Ref. [38]. 


\section{Code description}

MOLGW is being developed mainly in the Fortran 2003 language. In general, MOLGW prioritizes simplicity of implementation to facilitate new developments. As a consequence, symmetry operations are not used. All the Coulomb integrals are calculated once and then stored in memory (a so-called in-core algorithm). MOLGW's reliance on several external libraries eases the code's maintenance. 2-, 3-, and 4-center Coulomb integrals are all obtained from the library LIBINT [59], while the exchange-correlation energy density, potential, and kernel are calculated with the library LIBXC [62]. MOLGW is parallelized using Message Passing Interface (MPI) for the communications and ScaLAPACK for the linear algebra.

The accuracy of MOLGW for the self-consistent field has been tested against existing quantum chemistry codes with total energies and one-electron energies that agree within $10^{-6}$ Ha. Recently, we have also tested the accuracy against the FIESTA code for the $G W$ quasiparticle energies [87]. The agreement is better than $1 \mathrm{meV}$.

Regression tests are present that certify about 150 critical check points using 30 different input files.

Many basis sets are available with MoLGw. However, new ones can be obtained, for instance from the EMSL basis set exchange website $[88,89,90]$. A script is available in MOLGW that transforms the basis sets in NWCHEM [91] format into the internal format of MOLGW.

\section{Accuracy tests}

\subsection{Auxiliary basis set convergence}

Whereas the RI approximation has been assessed for MP2 calculations [93], its quality for $G W$ calculations is still rather unclear. While the RI for $G W$ has been used by several groups [94, 15, 41], MoLGW offers a unique opportunity to test this approximation precisely, since it is capable of turning it on or off.

As the $G W$ calculations without RI quickly become unfeasible, we have tested the approximation on a small molecule, namely silane $\mathrm{SiH}_{4}$. Silane is a relevant test example since the natures of the HOMO and LUMO differ significantly: the LUMO is unbound. In Table 1, we provide the results for the Dunning family of basis sets with and without diffuse functions $[95,96]$ in conjunction with the auxiliary basis set developed by Weigend and coworkers [92]. The error induced by the RI approximation appears as insensitive to the state nature (bound or unbound) as to the presence of diffuse function (augmented or not). The error is at most $11 \mathrm{meV}$,

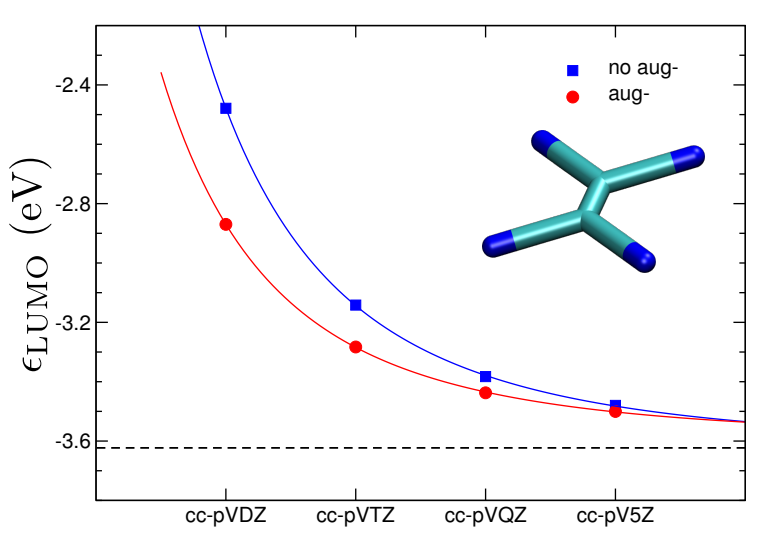

Figure 4: Basis set convergence of the LUMO energy of tetracyanoethylene (TCNE) with the Dunning basis sets without diffuse functions (blue squares) or with diffuse functions (red circles). The continuous curves are a fit to the calculated data with the function given in Eq. (54).

which is excellent compared to the other sources of error, such as the wavefunction basis set. We note that the RI error seems to decrease when going to more complete basis sets.

In our experience, the accuracy of the RI approximation shown here is typical of other small molecules. We conclude that the RI approximation can be safely used for production. It is used in all further shown calculations.

\subsection{Basis set convergence}

In several previous works [18, 39], we have already illustrated slow convergence of the $G W$ quasiparticle energies as a function of the basis set. We have shown that the slow convergence is not unexpected for a correlated method and the $G W$ convergence rate is not very different between MP2 and the coupled-cluster method $\operatorname{CCSD}(\mathrm{T})$.

Figure 4 shows a typical example of convergence for the famous-as-an-electron-acceptor tetracyanoethylene molecule (TCNE). We use the Dunning basis set families (cc-pVnZ and aug-cc-pVnZ) [95, 96] that are precisely designed to smoothly reach the complete basis set. The convergence is indeed smooth, but very slow. We propose an extrapolating function with 2 parameters $a, \epsilon_{m}^{\infty}$ for the quasiparticle energy as a function of the basis set,

$$
\epsilon_{m}\left(l_{\max }\right)=\epsilon_{m}^{\infty}+\frac{a}{l_{\max }^{2}},
$$


Table 1: HOMO and LUMO quasiparticle energy within $G W @ P B E 0$ for silane $\mathrm{SiH}_{4}$ for different Dunning basis sets using or not the RI approximation in $\mathrm{eV}$. The difference is given in $\mathrm{meV}$. We use here the auxiliary basis sets developed Weigend [92].

\begin{tabular}{lrcccccc}
\hline \hline \multirow{2}{*}{ HOMO } & & cc-pVDZ & cc-pVTZ & cc-pVQZ & aug-cc-pVDZ & aug-cc-pVTZ & aug-cc-pVQZ \\
& no RI (eV) & -12.3566 & -12.6027 & -12.6879 & -12.3426 & -12.6018 & -12.6958 \\
& RI (eV) & -12.3553 & -12.6012 & -12.6871 & -12.3407 & -12.6002 & -12.6952 \\
& Diff. $(\mathrm{meV})$ & 1.3 & 1.5 & 0.8 & 1.9 & 1.6 & 0.6 \\
\multirow{2}{*}{ LUMO } & & & & & & 0.6 \\
& no RI (eV) & 3.9687 & 3.2614 & 2.6863 & 0.9958 & 0.8431 & 0.7516 \\
& RI (eV) & 3.9800 & 3.2578 & 2.6864 & 0.9999 & 0.8420 & 0.7516 \\
& Diff. (meV) & 11.3 & -3.6 & 0.0 & 4.1 & -1.1 & -0.1 \\
\hline \hline
\end{tabular}

where $l_{\max }$ is the highest angular momentum in the basis set (cc-pVDZ has $l_{\max }=2$, cc-pVTZ has $l_{\max }=3$, etc.). The form of the extrapolating function has been empirically selected. The squared exponent in the denominator yields the best match with the explicitly-calculated points. Note that Truhlar [97] obtained a rather close exponent of 2.2 for the MP2 correlation energies. With this function, we have obtained the complete basis set value and hence we can define the basis set error. The error is as large as $1.1 \mathrm{eV}$ for cc-pVDZ and even the most complete basis set available, i.e. aug-cc-pV5Z, still experiences a $0.15 \mathrm{eV}$ deviation.

From all the examples we have calculated so far, the convergence of the quasiparticle energies as a function of the basis set size is always from above. As the convergence is indeed very slow, we advocate for the systematic use of an extrapolation technique when comparison to experiment is made.

We also provide the example of the convergence of an optical spectrum in Fig. 5. It shows the photoabsorption cross section of pyrene, $\mathrm{C}_{16} \mathrm{H}_{10}$, for different basis sets with and without diffuse functions. The whole calculation workflow (PBE0, then $G W$, then BSE) uses the same basis set. In contrast with the previous statement for $G W$, here the optical excitations converge smoothly with the basis set, especially for augmented basis sets. This demonstrates that the slow convergence of the quasiparticle energies observed above compensates, so that their differences converge more quickly. For instance, the first bright excitation at $3.42 \mathrm{eV}$ is evaluated with an accuracy of $50 \mathrm{meV}$ already with the augcc-pDZ basis. Just the higher energy lineshape around $7-9 \mathrm{eV}$ seems to be more sensitive to the basis set.

In conclusion, the convergence of the optical spectrum can in cases be achieved with unconverged absolute quasiparticle energies.

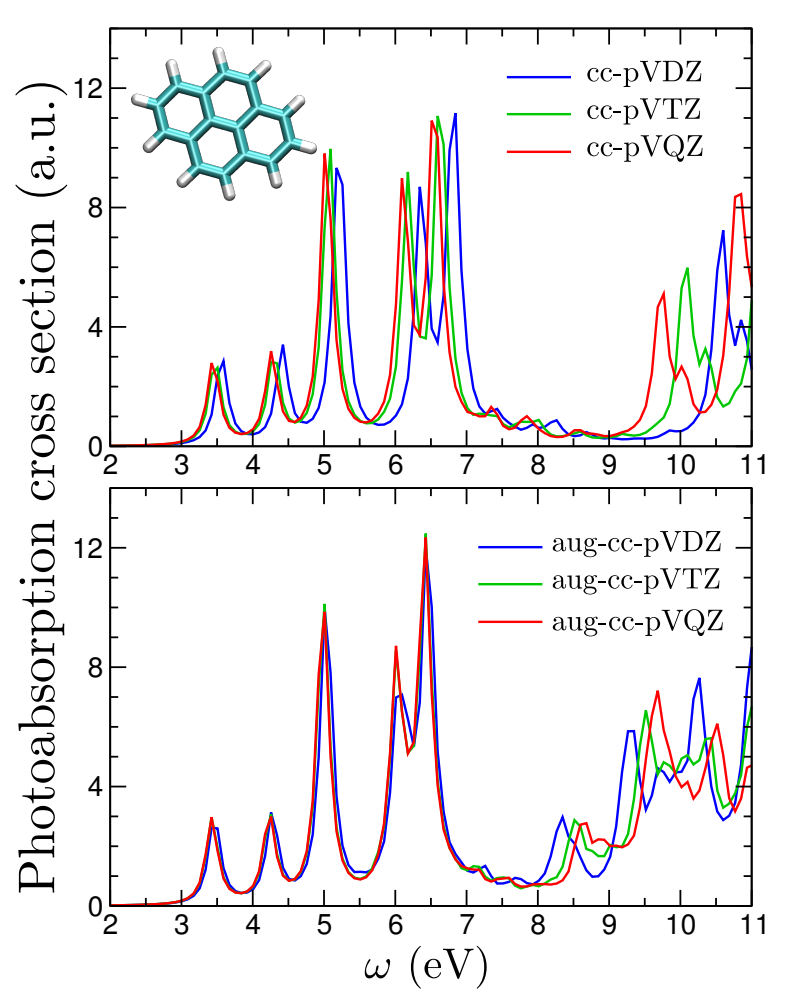

Figure 5: Basis set convergence of the photoabsorption cross section $\sigma$ of pyrene, $\mathrm{C}_{16} \mathrm{H}_{10}$, within BSE with with the Dunning basis sets without diffuse functions (upper panel) or with diffuse functions (lower panel). This calculation is based on PBE0. 

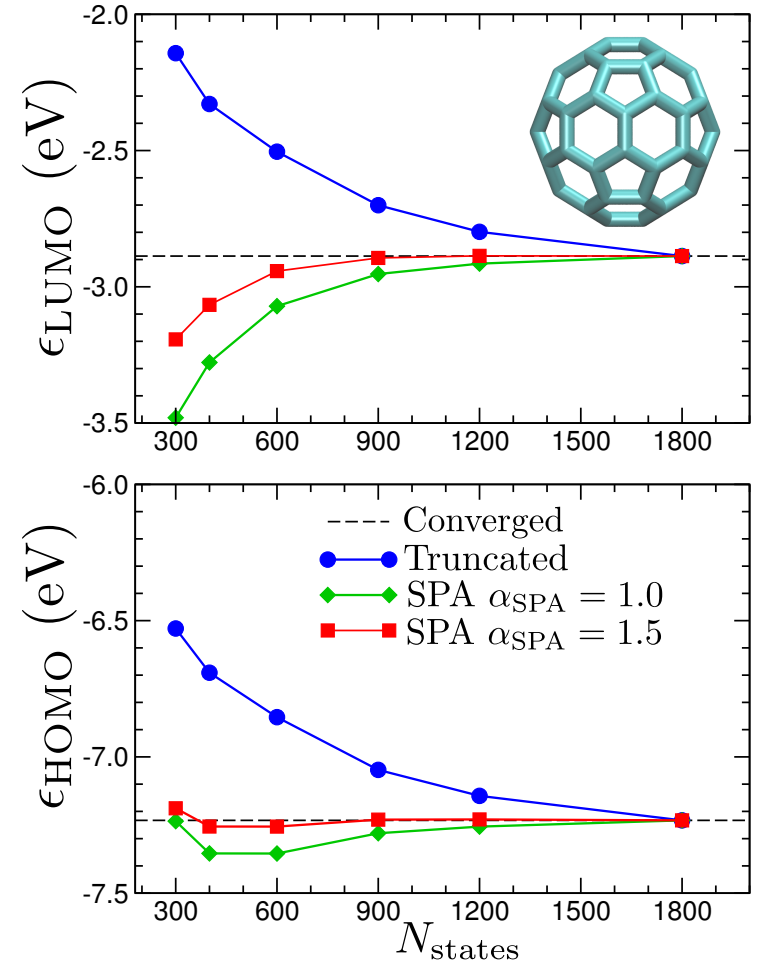

Figure 6: Convergence of the $G W @$ PBE0 HOMO and LUMO quasiparticle energies as a function of the number of states explicitely used in the calculatation of the RPA polarizability.

\subsection{Single Pole Approximation}

The strategy retained to evaluate the polarizability in MOLGW possesses the unique feature of being exact in the chosen basis set. Its drawback is that it scales poorly with system size. Indeed, the transition space is spanned by the product of orbitals $|i a \sigma\rangle$, where $i$ is occupied and $a$ is empty. This space scales as $N^{2}$, if $N$ is a measure of the system size (for instance the number of electrons). Then the matrix $C$ to be diagonalized in Eq. (41) occupies memory as $N^{4}$ and the diagonalization time itself scales as $N^{6}$. For large systems this can indeed be problematic. Consider buckminster fullerene $\mathrm{C}_{60}$ in the cc-pVTZ basis set. There are 1800 basis functions and, among them, 120 constitute the occupied states (considering that the $\mathrm{C} 1 s$ are frozen). The $C$ matrix is then a $194400 \times 194400$ real symmetric matrix that requires $281 \mathrm{~Tb}$ of storage. It is then necessary to have a procedure to reduce the size of the matrix while keeping reasonable accuracy.

A simple truncation of the number of empty states in the state product basis $|i a \sigma\rangle$ is not much help. We exemplify this statement with the convergence behav- ior of the HOMO and LUMO GW@PBE0 quasiparticle energies as shown in Fig. 6. When the product basis is limited to the empty states below a given state in$\operatorname{dex} N_{\text {states }}$, both the HOMO and LUMO converge very slowly to the value when all the empty states are included $\left(N_{\text {states }}=1800\right) . N_{\text {states }}=300$ yields quasiparticle energies that deviate by $0.7 \mathrm{eV}$ from the converged values within this basis set.

In the recent past, several techniques have been designed to account for the states that are neglected in the context of plane-wave approaches $[13,98,99]$. We propose to introduce the single pole approximation (SPA) [73] for the high energy states to reduce the $C$ matrix dimension. In this approximation, the RPA matrix elements when $a>N_{\text {states }}$ are considered diagonal:

$$
\begin{aligned}
& A_{i a \sigma}^{j b \sigma^{\prime}} \approx \delta_{i j} \delta_{a b} \delta_{\sigma \sigma^{\prime}}\left[\epsilon_{a}^{\sigma}-\epsilon_{i}^{\sigma}-\left(i a \sigma \mid i a \sigma^{\prime}\right)\right] \alpha_{\mathrm{SPA}} \\
& B_{i a \sigma}^{j b \sigma^{\prime}} \approx-\delta_{i j} \delta_{a b} \delta_{\sigma \sigma^{\prime}}\left(i a \sigma \mid a i \sigma^{\prime}\right) \alpha_{\mathrm{SPA}}
\end{aligned}
$$

Thus, the $C$ matrix has a dense square block up to $a=$ $N_{\text {states }}$ and is diagonal above. Then, the diagonalization needs only to be done for the dense block.

The performance of this procedure is shown with the green line in Fig. 6. The approximation clearly overshoots the targeted value for both the HOMO and the LUMO. As a consequence, we propose that the diagonal terms above $N_{\text {states }}$ be multiplied by an empirical parameter $\alpha_{\mathrm{SPA}}=1.5$. This empirical factor does not contain much physical meaning and it is just meant to reduce the overshooting of the SPA. However, it yields a substantial accuracy gain in the case of the $\mathrm{C}_{60}$ molecule, as can be observed from the red line in Fig. 6. Now an accuracy of $0.05 \mathrm{eV}$ can be reached for both the HOMO and the LUMO with $N_{\text {states }}=600$, therefore reducing the matrix dimension by a factor of 4 and hence dividing the computational time by 64 ! This parameter $\alpha_{\text {SPA }}$ is meant to be tuned, however the values ranging from 1.0 to 1.5 are quite reasonable across all the molecules we have calculated so far.

The use of the SPA allows one to significantly reduce computational time and memory consumption by changing the scaling prefactor, however keeping an overall $N^{6}$ scaling.

\section{Performance and parallelization}

To access the $G W$ quasiparticle energies and the BSE optical spectra of larger molecules, the parallelization of the code becomes unavoidable. Since the medium to large molecules can be calculated within a reasonable 

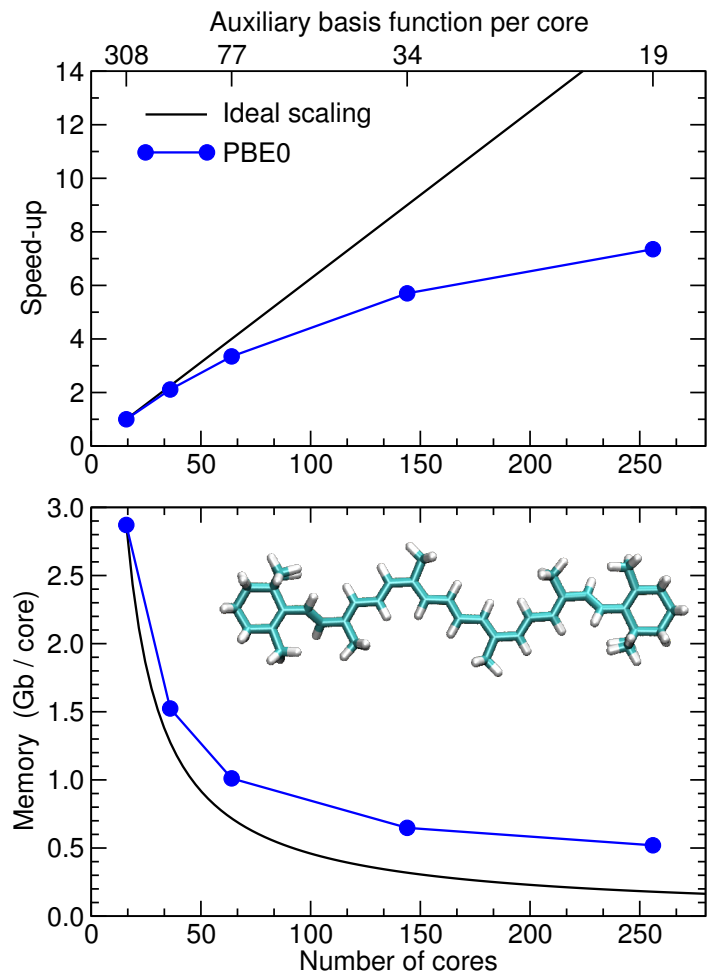

Figure 7: Parallelization performance of the gKS part of MoLGw for the $\beta$-Caroten molecule $\mathrm{C}_{40} \mathrm{H}_{56}$ within the rather complete basis set cc-pVTZ. The upper panel represents the computational time speedup as a function of the number of cores. The reference for speed-up is taken for 16 cores. The lower panel shows the memory consumption per core. The actual results (blue circles) are compared to the ideal parallelization (black line).

time only when the RI approximation is used, the parallelization effort has been focusing on the RI calculations in MoLGw. Two types of operations benefit from the parallelization: the construction of the operators, such as the Hamiltonian in Eq. (16) or the polarizability matrices $A$ and $B$ in Eqs. (37a), (37b), and the large eigenvalue problem described in Eq. (36).

\subsection{Parallelization for the construction of the opera- tors}

As the parallelization is only meant to be used in conjunction with the RI approximation, the parallelization of the data and of the computation tasks most often relies on the distribution over the auxiliary basis functions $P$. This choice is validated by the observation that the number of basis functions $P$ in the auxiliary basis is in general much larger than the number of functions in the wave function basis $\mu$. Furthermore, distributing over the auxiliary basis functions allows us to calculate many operators with only a few changes from the serial version of the code.

Let us exemplify the parallelization with the calculation of the exchange operator $K$. The auxiliary basis functions $P$ are divided among the processors. Except for a subset of $P$, each processor owns in memory the 3-center Coulomb integral $M_{\mu \nu}^{P}$ for all $\mu$ and $v$. Upon introduction of the RI approximation [See Eq. (31)], and the square root of the density matrix $P_{\mu \nu}^{\sigma}$, Eq. (22) can be cast into

$$
K_{\mu \nu}^{\sigma}=\sum_{P} \sum_{\kappa}\left(\sum_{\lambda} M_{\mu \lambda}^{P} P_{\lambda \kappa}^{\sigma 1 / 2}\right)\left(\sum_{\tau} M_{\nu \tau}^{P} P_{\tau \kappa}^{\sigma 1 / 2}\right),
$$

where $\kappa$ only runs over the non-vanishing eigenvalues of $P$, i.e. the occupied states. As can be observed from Eq. (56), the sum over $P$ is only performed at the end of the calculation of $K_{\mu \nu}^{\sigma}$. Therefore, each processor can calculate its workload independently and only at the end, an overall reduction is carried out.

Another example is that of the well-known atomic orbital to molecular orbital transforms that are necessary to evaluate the integrals ( $\left.i a \sigma \mid j b \sigma^{\prime}\right)$ in Eqs. (37a), (37b). In this case, the parallelization is completely independent. Each processor performs the transforms

$$
M_{m n \sigma}^{P}=\sum_{\mu v} C_{\mu m}^{\sigma} C_{v n}^{\sigma} M_{\mu v}^{P}
$$

for its subset of auxiliary basis functions $P$. Then the integrals $\left(i a \sigma \mid j b \sigma^{\prime}\right)$ are calculated following the same spirit as in Eq. (56).

In Fig. 7, we show the performance of the parallelization of MoLGW for the gKS part for a medium molecule with almost 100 atoms, namely $\beta$-Carotene $\mathrm{C}_{40} \mathrm{H}_{56} \cdot \beta$ Carotene using the cc-pVTZ basis set has 1984 basis functions. The corresponding auxiliary basis has 4920 functions. The speed-up has been calculated with respect to the smallest core number that fits in memory, i.e. 16 cores. The scaling of the computational time is rather good as long as the number of auxiliary basis functions per core is not too low, as demonstrated in the upper panel of Fig. 7. A lower limit of 30-50 auxiliary basis functions per core is reasonable. It should be stressed that anyway, the computational time is not large: even with just 16 cores, the calculation lasts only roughly 3 hours.

Concerning the memory consumption shown in the lower panel of Fig. 7, the parallelization of the data is efficient. This is not surprizing since, for the in-core algorithm used in MOLGW, most of the memory is used to store the 3-center Coulomb integrals $M_{\mu \nu}^{P}$ that are perfectly distributed among the cores. 
In general, the bottleneck of the calculation is not the gKS step. Rather, it is the calculation of the polarizability, either to prepare the $G W$ self-energy evaluation or to evaluate the optical spectrum within BSE.

\subsection{Diagonalizing the BSE Hamiltonian in parallel}

Within the semi-local approximations to TD-DFT and within RPA, the matrix $(A-B)$ entering the expression of $C$ in Eq. (41) is purely diagonal and therefore its square-root can straightforwardly be calculated.

On the contrary, for hybrid functionals or for BSE, the matrix $(A-B)$ is dense and the direct application of Eq. (41) would require two diagonalizations. To avoid this, we use the Cholesky decomposition to replace the matrix square root. Our approach is summarized as follows [100]:

1. Compute the Cholesky factorization $A+B=L L^{T}$.

2. Form $W=L^{T}(A-B) L$.

3. Diagonalize $W=Z \Omega Z^{T}$.

4. Set $X=\left(L^{-T} Z \Omega^{1 / 2}+L Z \Omega^{1 / 2}\right) / 2$.

5. Set $Y=\left(L^{-T} Z \Omega^{1 / 2}-L Z \Omega^{1 / 2}\right) / 2$.

All of these steps make use of existing functionalities from the ScaLAPACK software package [101]. The eigenvectors are normalized as $X^{T} X-Y^{T} Y=I$ so that both right and left eigenvectors can be readily used without further scaling.

Figure 8 exemplifies the performance of the BSE solver in MOLGW with an oligomer, namely polyacetylene $\mathrm{C}_{2 n} \mathrm{H}_{2 n+2}$. The scaling with system size is made apparent. The number of transitions from occupied to empty states scales as $n^{2}$. The BSE matrix is $n^{2} \times n^{2}$. The time necessary to construct it (upper panel) and the memory necessary to store it (lower panel) indeed scale as $n^{4}$. Then the structure preserving algorithm presented above is dominated by the diagonalization in its step 3 . This operation scales as the cube of the matrix dimension, which yields an overall scaling of $n^{6}$. The measured slopes in Fig. 8 match rather well with the scalings we have just anticipated.

As the calculation complexity increases quickly with the size of the problem, parallelization of the code is highly necessary. MOLGW uses a ScaLAPACK distribution of the BSE matrix to distribute the memory and workload for the diagonalization. It can be observed from the lower panel of Fig. 8 that by increasing the number of cores the memory consumption can be kept below $2 \mathrm{~Gb}$ per core, which is a reasonable target for modern computers. The middle panel shows how well the structure preserving algorithm behaves as a function of the number of cores. The construction of the BSE
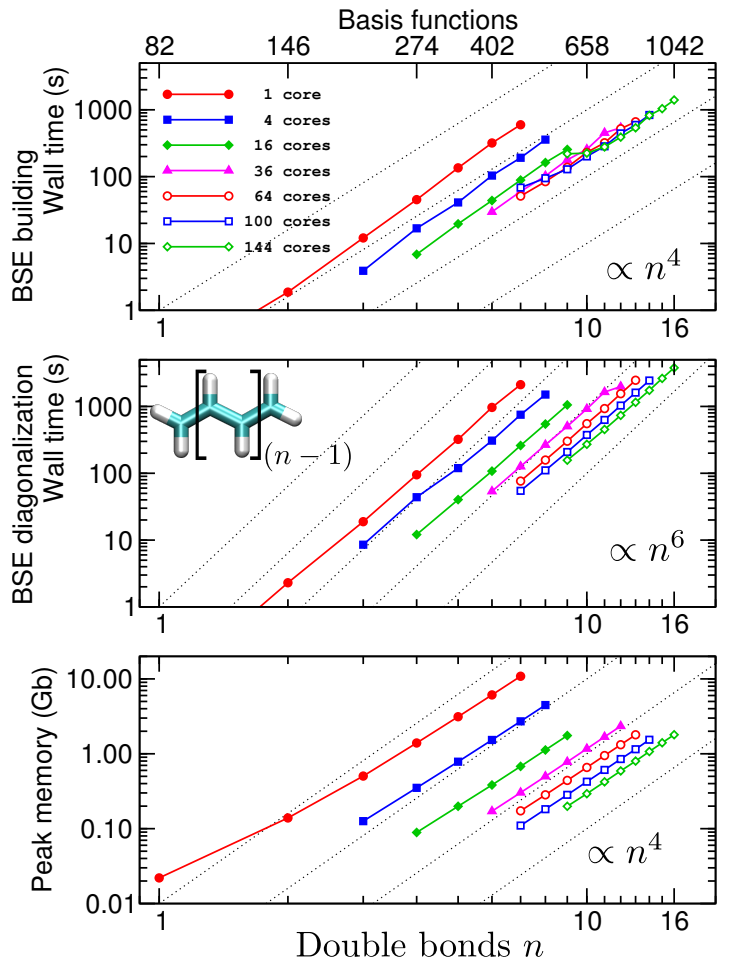

Figure 8: Numerical performance of the BSE solution in MOLGW for polyacethylene oligomer $\mathrm{C}_{2 n} \mathrm{H}_{2 n+2}$ within the aug-cc-pVDZ basis with increasing length $n$. The upper panel shows the wall clock time necessary to build the BSE. The middle panel shows the wall clock time used in the structure preserving solution of the BSE. The lower panel shows the peak memory used in a BSE run. The thin dotted lines highlight the anticipated scaling of the computational load. The calculations have been performed with a number of cores ranging from 1 to 144 . 


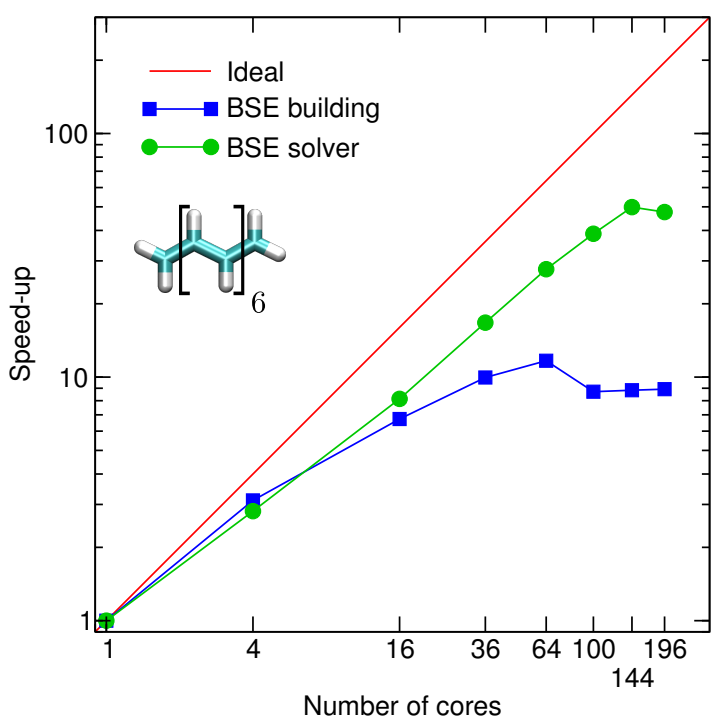

Figure 9: Parallelization speed-up of a BSE calculation for a small polyacethylene oligomer $\mathrm{C}_{14} \mathrm{H}_{16}$ within the aug-cc-pVDZ basis as a function of the number of cores. The BSE calculation timing is split into the building-up of the BSE matrix (blue squares) and its structure preserving diagonalization (green circles).

matrix in the upper panel is more problematic for the parallelization. Indeed, as in the rest of the code, the calculation of the BSE matrix elements relies on the distribution of the auxiliary basis. Then this distribution of the data must be transposed into the ScaLAPACK distribution, which induces some intensive communications.

The performance as a function of the number of cores is perhaps better appreciated from Fig. 9. For this small example, we have managed to multiply the number of cores up to the parallelization breakdown. The BSE matrix building experiences an efficiency collapse beyond 64 cores, whereas the structure preserving diagonalization based on ScaLAPACK allows one to reach 144 cores without breakdown. It is remarkable that the speed-up is rather close to the ideal one over two orders of magnitude of core counts.

Of course, MOLGW offers the possibility to limit the BSE matrix size by removing the highest empty states from the transition basis. This approximation should be tested as an extra convergence parameter.

\section{Summary}

In this paper, we have presented version 1 of the MOLGW code. This code is dedicated to MBPT calculations in the $G W$ approximation for atoms, molecules, and clusters. The focus is to calculate i) the $G W$ selfenergy to obtain accurate quasiparticle energies that may be compared to photoemission spectroscopy and ii) the solutions to the BSE, to evaluate precise optical spectra that include the electron-hole interaction. MOLGW relies on Gaussian type orbitals and therefore make use of all of the past developments in quantum chemistry.

We have shown the most central equations used in practice in MOLGW, starting from the self-consistent field within $\mathrm{gKS}$, to the $G W$ self-energy and BSE solution. MOLGW is a unique code, since it implements the equations with or without the RI approximation. It also has the advantage of calculating directly the spectral decomposition of the screened Coulomb interaction $W$ and hence is able to calculate the $G W$ self-energy at any frequency $\omega$ with no further technical approximation. Incidentally, the RPA total energy is obtained for free. MOLGW employs a recently developed structure preserving parallel algorithm to perform the diagonalization of the BSE Hamiltonian.

This paper was also an opportunity to demonstrate the validity of the RI by directly comparing calculations with and without this approximation. Furthermore, we introduced a technique, based on the single pole approximation, to speed-up the $G W$ calculations and save memory.

In conclusion, we stress that MOLGW version 1 is enclosed with this paper. However the forthcoming updates will be directly and freely obtained on a versioning server hosted on the web [102].

\section{Acknowledgement}

F.B. acknowledges the Enhanced Eurotalent program and the France Berkeley Fund for supporting his sabbatical leave in UC Berkeley. This work is supported by the U.S. Department of Energy, Office of Basic Energy Sciences and of Advanced Scientific Computing Research through the SciDAC Program on Excited State Phenomena. S.M.H. is supported by the Chemical Sciences, Geosciences, and Biosciences Division in Office of Basic Energy Sciences of the U.S. Department of Energy. Portions of this work took place at the Molecular Foundry, supported by the U.S. Department of Energy, Office of Basic Energy Sciences. This work was performed using HPC resources from GENCI-CCRTTGCC (Grants No. 2015-096018).

[1] A. L. Fetter, J. D. Walecka, Quantum theory of Many-Particle Systems, MacGraw-Hill, New York, 1971.

[2] G. D. Mahan, Many-Particle Physics, 3rd Edition, Kluwer Academic/Plenum Publishers, 2000. 
[3] A. Szabó, N. S. Ostlund, Modern quantum chemistry : introduction to advanced electronic structure theory, Dover Publications, Mineola (N.Y.), 1996.

[4] C. Møller, M. S. Plesset, Note on an approximation treatment for many-electron systems, Phys. Rev. 46 (1934) 618-622. doi:10.1103/PhysRev.46.618.

[5] L. Hedin, New method for calculating the one-particle green's function with application to the electron-gas problem, Phys. Rev. 139 (1965) A796-A823. doi:10.1103/PhysRev.139.A796.

[6] G. Strinati, H. J. Mattausch, W. Hanke, Dynamical correlation effects on the quasiparticle bloch states of a covalent crystal, Phys. Rev. Lett. 45 (1980) 290-294. doi:10.1103/PhysRevLett.45.290.

[7] M. S. Hybertsen, S. G. Louie, First-principles theory of quasiparticles: Calculation of band gaps in semiconductors and insulators, Phys. Rev. Lett. 55 (1985) 1418-1421. doi:10.1103/PhysRevLett.55.1418.

[8] F. Aryasetiawan, O. Gunnarsson, The $G W$ method, Rep. Prog. Phys. 61 (1998) 237-312. doi:10.1088/0034-4885/61/3/002.

[9] F. Bruneval, M. Gatti, Quasiparticle self-consistent $G W$ method for the spectral properties of complex materials, in: C. Di Valentin, S. Botti, M. Cococcioni (Eds.), First Principles Approaches to Spectroscopic Properties of Complex Materials, Topics in Current Chemistry, Springer Berlin Heidelberg, 2014, pp. 1-37. doi:10.1007/128_2013_460.

[10] E. L. Shirley, R. M. Martin, $G W$ quasiparticle calculations in atoms, Phys. Rev. B 47 (1993) 15404-15412. doi:10.1103/PhysRevB.47.15404.

[11] J. C. Grossman, M. Rohlfing, L. Mitas, S. G. Louie, M. L. Cohen, High accuracy many-body calculational approaches for excitations in molecules, Phys. Rev. Lett. 86 (2001) 472-475. doi:10.1103/PhysRevLett.86.472.

[12] N. E. Dahlen, U. v. Barth, Variational energy functionals tested on atoms, Phys. Rev. B 69 (2004) 195102. doi:10.1103/PhysRevB.69.195102.

[13] M. L. Tiago, J. R. Chelikowsky, Optical excitations in organic molecules, clusters, and defects studied by first-principles greens function methods, Phys. Rev. B 73 (2006) 205334. doi:10.1103/PhysRevB.73.205334.

[14] C. Rostgaard, K. W. Jacobsen, K. S. Thygesen, Fully selfconsistent gw calculations for molecules, Phys. Rev. B 81 (2010) 085103. doi:10.1103/PhysRevB.81.085103.

[15] X. Blase, C. Attaccalite, V. Olevano, First-principles $G W$ calculations for fullerenes, porphyrins, phtalocyanine, and other molecules of interest for organic photovoltaic applications, Phys. Rev. B 83 (2011) 115103. doi:10.1103/PhysRevB.83.115103.

[16] D. Foerster, P. Koval, D. S' anchez Portal, An o(n3) implementation of hedinś gw approximation for molecules, J. Chem. Phys. 135 (7) (2011) 074105. doi:http://dx.doi.org/10.1063/1.3624731.

[17] A. Ferretti, G. Mallia, L. Martin-Samos, G. Bussi, A. Ruini, B. Montanari, N. M. Harrison, Ab initio complex band structure of conjugated polymers: Effects of hydrid density functional theory and $G W$ schemes, Phys. Rev. B 85 (2012) 235105. doi:10.1103/PhysRevB.85.235105.

[18] F. Bruneval, Ionization energy of atoms obtained from $G W$ self-energy or from random phase approximation total energies, J. Chem. Phys. 136 (19) (2012) 194107. doi:http://dx.doi.org/10.1063/1.4718428.

[19] N. Marom, F. Caruso, X. Ren, O. T. Hofmann, T. Körzdörfer, J. R. Chelikowsky, A. Rubio, M. Scheffler, P. Rinke, Benchmark of $g w$ methods for azabenzenes, Phys. Rev. B 86 (2012) 245127. doi:10.1103/PhysRevB.86.245127.

[20] T. Körzdörfer, N. Marom, Strategy for finding a reliable start- ing point for $G_{0} W_{0}$ demonstrated for molecules, Phys. Rev. B 86 (2012) 041110. doi:10.1103/PhysRevB.86.041110.

[21] F. Caruso, P. Rinke, X. Ren, M. Scheffler, A. Rubio, Unified description of ground and excited states of finite systems: The self-consistent $g w$ approach, Phys. Rev. B 86 (2012) 081102. doi:10.1103/PhysRevB.86.081102.

[22] F. Caruso, D. R. Rohr, M. Hellgren, X. Ren, P. Rinke, A. Rubio, M. Scheffler, Bond breaking and bond formation: How electron correlation is captured in many-body perturbation theory and density-functional theory, Phys. Rev. Lett. 110 (2013) 146403. doi:10.1103/PhysRevLett.110.146403.

[23] P. Koval, D. Foerster, D. Sánchez-Portal, Fully self-consistent $g w$ and quasiparticle self-consistent $g w$ for molecules, Phys. Rev. B 89 (2014) 155417. doi:10.1103/PhysRevB.89.155417.

[24] A. Hauschild, K. Karki, B. C. C. Cowie, M. Rohlfing, F. S. Tautz, M. Sokolowski, Molecular distortions and chemical bonding of a large $\pi$-conjugated molecule on a metal surface, Phys. Rev. Lett. 94 (2005) 036106. doi:10.1103/PhysRevLett.94.036106.

[25] J. B. Neaton, M. S. Hybertsen, S. G. Louie, Renormalization of molecular electronic levels at metalmolecule interfaces, Phys. Rev. Lett. 97 (2006) 216405. doi:10.1103/PhysRevLett.97.216405.

[26] S. Y. Quek, H. J. Choi, S. G. Louie, J. B. Neaton, Length dependence of conductance in aromatic singlemolecule junctions, Nano Letters 9 (11) (2009) 39493953. arXiv:http://dx.doi.org/10.1021/n19021336, doi:10.1021/n19021336.

[27] T. Rangel, A. Ferretti, P. E. Trevisanutto, V. Olevano, G.M. Rignanese, Transport properties of molecular junctions from many-body perturbation theory, Phys. Rev. B 84 (2011) 045426. doi:10.1103/PhysRevB.84.045426.

[28] M. Strange, C. Rostgaard, H. Häkkinen, K. S. Thygesen, Selfconsistent gw calculations of electronic transport in thiol- and amine-linked molecular junctions, Phys. Rev. B 83 (2011) 115108. doi:10.1103/PhysRevB.83.115108.

[29] X. Gonze, B. Amadon, P. M. Anglade, J. M. Beuken, F. Bottin, P. Boulanger, F. Bruneval, D. Caliste, R. Caracas, M. Côté, T. Deutsch, L. Genovese, P. Ghosez, M. Giantomassi, S. Goedecker, D. R. Hamann, P. Hermet, F. Jollet, G. Jomard, S. Leroux, M. Mancini, S. Mazevet, M. J. T. Oliveira, G. Onida, Y. Pouillon, T. Rangel, G. M. Rignanese, D. Sangalli, R. Shaltaf, M. Torrent, M. J. Verstraete, G. Zerah, J. W. Zwanziger, Abinit: First-principles approach to material and nanosystem properties, Comput. Phys. Commun. 180 (2009) 2582. doi:http://dx.doi.org/10.1016/j.cpc.2009.07.007.

[30] A. Marini, C. Hogan, M. Grning, D. Varsano, yambo: An ab initio tool for excited state calculations, Comput. Phys. Commun. 180 (8) (2009) 1392 - 1403. doi:http://dx.doi.org/10.1016/j.cpc.2009.02.003.

[31] L. Martin-Samos, G. Bussi, Sax: An open source package for electronic-structure and optical-properties calculations in the $\{\mathrm{GW}\}$ approximation, Comput. Phys. Commun. 180 (8) (2009) 1416 - 1425. doi:http://dx.doi.org/10.1016/j.cpc.2009.02.005.

[32] M. Shishkin, G. Kresse, Implementation and performance of the frequency-dependent $g w$ method within the paw framework, Phys. Rev. B 74 (2006) 035101. doi:10.1103/PhysRevB.74.035101.

[33] J. Deslippe, G. Samsonidze, D. A. Strubbe, M. Jain, M. L. Cohen, S. G. Louie, Berkeleygw: A massively parallel computer package for the calculation of the quasiparticle and optical properties of materials and nanostructures, Comput. Phys. Commun. 183 (6) (2012) 1269 - 1289. doi:http://dx.doi.org/10.1016/j.cpc.2011.12.006.

[34] P. Umari, G. Stenuit, S. Baroni, Gw quasiparticle spectra 
from occupied states only, Phys. Rev. B 81 (2010) 115104. doi:10.1103/PhysRevB.81.115104.

[35] M. Govoni, G. Galli, Large scale gw calculations, J. Chem. Theory Comput. 11 (6) (2015) 2680-2696, pMID: 26575564. arXiv:http://dx.doi.org/10.1021/ct500958p, doi:10.1021/ct500958p.

[36] V. Blum, R. Gehrke, F. Hanke, P. Havu, V. Havu, $\mathrm{X}$. Ren, K. Reuter, M. Scheffler, Ab initio molecular simulations with numeric atom-centered orbitals, Comput. Phys. Commun. 180 (11) (2009) 2175 - 2196. doi:http://dx.doi.org/10.1016/j.cpc.2009.06.022.

[37] M. J. van Setten, F. Weigend, F. Evers, The gw-method for quantum chemistry applications: Theory and implementation, J. Chem. Theory Comput. 9 (1) (2013) 232-246, pMID: 26589026. arXiv:http://dx.doi.org/10.1021/ct300648t, doi:10.1021/ct300648t.

[38] F. Bruneval, S. M. Hamed, J. B. Neaton, A systematic benchmark of the ab initio bethe-salpeter equation approach for low-lying optical excitations of small organic molecules, J. Chem. Phys. 142 (24) (2015) 244101. doi:http://dx.doi.org/10.1063/1.4922489.

[39] F. Bruneval, M. A. L. Marques, Benchmarking the starting points of the gw approximation for molecules, J. Chem. Theory Comput. 9 (1) (2013) 324-329. arXiv:http://pubs.acs.org/doi/pdf/10.1021/ct300835h, doi: $10.1021 / \operatorname{ct} 300835 \mathrm{~h}$.

[40] M. P. Ljungberg, P. Koval, F. Ferrari, D. Foerster, D. SánchezPortal, Cubic-scaling iterative solution of the bethe-salpeter equation for finite systems, Phys. Rev. B 92 (2015) 075422. doi:10.1103/PhysRevB.92.075422.

[41] X. Ren, P. Rinke, V. Blum, J. Wieferink, A. Tkatchenko, A. Sanfilippo, K. Reuter, M. Scheffler, Resolution-of-identity approach to hartreefock, hybrid density functionals, rpa, mp2 and gw with numeric atom-centered orbital basis functions, New Journal of Physics 14 (5) (2012) 053020.

[42] D. Jacquemin, I. Duchemin, X. Blase, Benchmarking the bethesalpeter formalism on a standard organic molecular set, J. Chem. Theory Comput. 11 (7) (2015) 32903304. arXiv:http://dx.doi.org/10.1021/acs.jctc.5b00304, doi:10.1021/acs.jctc.5b00304.

[43] D. Jacquemin, I. Duchemin, X. Blase, 00 energies using hybrid schemes: Benchmarks of td-dft, cis(d), $\operatorname{adc}(2)$, $\mathrm{cc} 2$, and bse/gw formalisms for 80 real-life compounds, J. Chem. Theory Comput. 11 (11) (2015) 5340-5359, pMID: 26574326. arXiv:http://dx.doi.org/10.1021/acs.jctc.5b00619, doi:10.1021/acs.jctc.5b00619.

[44] J. W. Knight, X. Wang, L. Gallandi, O. Dolgounitcheva, X. Ren, J. V. Ortiz, P. Rinke, T. Krzdrfer, N. Marom, Accurate ionization potentials and electron affinities of acceptor molecules iii: A benchmark of gw methods, Journal of Chemical Theory and Computation 0 (0) (0) null, pMID: 26731609. arXiv:http://dx.doi.org/10.1021/acs.jctc.5b00871, doi:10.1021/acs.jctc.5b00871.

[45] G. Strinati, Application of the greens-functions method to the study of the optical-properties of semiconductors, Riv. Nuovo Cimento 11 (1988) 1. doi:10.1007/BF02725962.

[46] G. Onida, L. Reining, A. Rubio, Electronic excitations: density-functional versus many-body green's-function approaches, Rev. Mod. Phys. 74 (2002) 601-659. doi:10.1103/RevModPhys.74.601.

[47] M. Shishkin, G. Kresse, Self-consistent $g w$ calculations for semiconductors and insulators, Phys. Rev. B 75 (2007) 235102. doi:10.1103/PhysRevB.75.235102.

[48] S. V. Faleev, M. van Schilfgaarde, T. Kotani, Allelectron self-consistent $g w$ approximation: Application to si, mno, and nio, Phys. Rev. Lett. 93 (2004) 126406. doi:10.1103/PhysRevLett.93.126406.

[49] M. van Schilfgaarde, T. Kotani, S. Faleev, Quasiparticle selfconsistent $G W$ theory, Phys. Rev. Lett. 96 (2006) 226402. doi:10.1103/PhysRevLett.96.226402.

[50] F. Bruneval, N. Vast, L. Reining, Effect of self-consistency on quasiparticles in solids, Phys. Rev. B 74 (2006) 045102. doi:10.1103/PhysRevB.74.045102.

[51] A. Stan, N. E. Dahlen, R. van Leeuwen, Fully self-consistent $G W$ calculations for atoms and molecules, Europhys. Lett. 76 (2006) 298. doi:10.1209/epl/i2006-10266-6.

[52] H. B. Schlegel, M. J. Frisch, Transformation between cartesian and pure spherical harmonic gaussians, Int. J. Quantum Chem. 54 (2) (1995) 83-87. doi:10.1002/qua.560540202.

[53] A. D. Becke, A new mixing of hartreefock and local densityfunctional theories, J. Chem. Phys. 98 (2) (1993) 1372-1377. doi:http://dx.doi.org/10.1063/1.464304.

[54] C. Adamo, V. Barone, Toward reliable density functional methods without adjustable parameters: The pbe0 model, J. Chem. Phys. 110 (13) (1999) 6158-6170. doi:http://dx.doi.org/10.1063/1.478522.

[55] S. Refaely-Abramson, R. Baer, L. Kronik, Fundamental and excitation gaps in molecules of relevance for organic photovoltaics from an optimally tuned rangeseparated hybrid functional, Phys. Rev. B 84 (2011) 075144. doi:10.1103/PhysRevB.84.075144.

[56] J. Heyd, G. E. Scuseria, M. Ernzerhof, Hybrid functionals based on a screened coulomb potential, J. Chem. Phys. 118 (18) (2003) 8207-8215. doi:http://dx.doi.org/10.1063/1.1564060.

[57] J. Heyd, G. E. Scuseria, M. Ernzerhof, Erratum: hybrid functionals based on a screened coulomb potential $[\mathrm{j}$. chem. phys.118, 8207 (2003)], J. Chem. Phys. 124 (21) (2006) 219906. doi:http://dx.doi.org/10.1063/1.2204597.

[58] S. Obara, A. Saika, Efficient recursive computation of molecular integrals over cartesian gaussian functions, J. Chem. Phys. 84 (7) (1986) 3963-3974. doi:http://dx.doi.org/10.1063/1.450106.

[59] E. F. Valeev, A library for the evaluation of molecular integrals of many-body operators over gaussian functions, http://libint.valeyev.net/ (2016).

[60] D. L. Strout, G. E. Scuseria, A quantitative study of the scaling properties of the hartreefock method, The Journal of Chemical Physics 102 (21) (1995) 8448-8452. doi:http://dx.doi.org/10.1063/1.468836.

[61] J. A. Pople, P. M. Gill, B. G. Johnson, Kohn-sham densityfunctional theory within a finite basis set, Chem. Phys. Lett. 199 (6) (1992) 557 - 560. doi:http://dx.doi.org/10.1016/00092614(92)85009-Y

[62] M. A. L. Marques, M. J. T. Oliveira, T. Burnus, Libxc: a library of exchange and correlation functionals for density functional theory, Comput. Phys. Commun. 183 (2012) 2272. doi:10.1016/j.cpc.2012.05.007.

[63] A. D. Becke, A multicenter numerical integration scheme for polyatomic molecules, J. Chem. Phys. 88 (4) (1988) 25472553. doi:http://dx.doi.org/10.1063/1.454033.

[64] R. Stratmann, G. E. Scuseria, M. J. Frisch, Achieving linear scaling in exchange-correlation density functional quadratures, Chem. Phys. Lett. 257 (1996) 213 - 223. doi:http://dx.doi.org/10.1016/0009-2614(96)00600-8.

[65] V. I. Lebedev, D. N. Laikov, A quadrature formula for the sphere of the 131st algebraic order of accuracy, Doklady Mathematics 59 (3) (1999) 477-481.

[66] M. E. Mura, P. J. Knowles, Improved radial grids for quadrature in molecular densityfunctional calcu- 
lations, J. Chem. Phys. 104 (24) (1996) 9848-9858. doi:http://dx.doi.org/10.1063/1.471749.

[67] P. Pulay, Convergence acceleration of iterative sequences. the case of scf iteration, Chem. Phys. Lett. 73 (2) (1980) 393 398. doi:http://dx.doi.org/10.1016/0009-2614(80)80396-4.

[68] P. Pulay, Improved scf convergence acceleration, J. Comput. Chem. 3 (4) (1982) 556-560. doi:10.1002/jcc.540030413.

[69] K. Eichkorn, O. Treutler, H. hm, M. Hser, R. Ahlrichs, Auxiliary basis sets to approximate coulomb potentials, Chem. Phys. Lett. 240 (4) (1995) 283 - 290. doi:http://dx.doi.org/10.1016/0009-2614(95)00621-A.

[70] K. Eichkorn, F. Weigend, O. Treutler, R. Ahlrichs, Auxiliary basis sets for main row atoms and transition metals and their use to approximate coulomb potentials, Theor. Chem. Acc. 97 (1-4) (1997) 119-124. doi:10.1007/s002140050244.

[71] F. Weigend, A fully direct ri-hf algorithm: Implementation, optimised auxiliary basis sets, demonstration of accuracy and efficiency, Phys. Chem. Chem. Phys. 4 (2002) 4285-4291. doi:10.1039/B204199P.

[72] A. D. McLachlan, M. A. Ball, Time-dependent hartree-fock theory for molecules, Rev. Mod. Phys. 36 (1964) 844-855. doi:10.1103/RevModPhys.36.844.

[73] C. A. Ullrich, Time-Dependent Density-Functional Theory: Concepts and Applications, Oxford Graduate Texts, Oxford University Press, Oxford, New York, 2012.

[74] M. E. Casida, Recent Advances in Density Functional Methods, Part I, World Scientific, Singapore, 1995, p. 155.

[75] B. Holm, Total energies from $G W$ calculations, Phys. Rev. Lett. 83 (1999) 788-791. doi:10.1103/PhysRevLett.83.788.

[76] N. E. Dahlen, R. van Leeuwen, U. von Barth, Variational energy functionals of the green function and of the density tested on molecules, Phys. Rev. A 73 (2006) 012511. doi:10.1103/PhysRevA.73.012511.

[77] A. Klein, Perturbation theory for an infinite medium of fermions. ii, Phys. Rev. 121 (1961) 950-956. doi:10.1103/PhysRev.121.950.

[78] J. F. Dobson, J. Wang, Successful test of a seamless van der waals density functional, Phys. Rev. Lett. 82 (1999) 21232126. doi:10.1103/PhysRevLett.82.2123.

[79] F. Furche, Molecular tests of the random phase approximation to the exchange-correlation energy functional, Phys. Rev. B 64 (2001) 195120. doi:10.1103/PhysRevB.64.195120.

[80] M. Fuchs, X. Gonze, Accurate density functionals: Approaches using the adiabatic-connection fluctuationdissipation theorem, Phys. Rev. B 65 (2002) 235109. doi:10.1103/PhysRevB.65.235109.

[81] F. Furche, Developing the random phase approximation into a practical post-kohnsham correlation model, J. Chem. Phys. 129 (11) (2008) 114105. doi:http://dx.doi.org/10.1063/1.2977789.

[82] M. S. Hybertsen, S. G. Louie, Electron correlation in semiconductors and insulators: Band gaps and quasiparticle energies, Phys. Rev. B 34 (1986) 5390-5413. doi:10.1103/PhysRevB.34.5390.

[83] W. Hanke, L. J. Sham, Local-field and excitonic effects in the optical spectrum of a covalent crystal, Phys. Rev. B 12 (1975) 4501-4511. doi:10.1103/PhysRevB.12.4501.

[84] S. Albrecht, L. Reining, R. Del Sole, G. Onida, Ab Initio calculation of excitonic effects in the optical spectra of semiconductors, Phys. Rev. Lett. 80 (1998) 4510-4513. doi:10.1103/PhysRevLett.80.4510.

[85] E. L. Shirley, $A b$ Initio inclusion of electron-hole attraction: Application to $\mathrm{x}$-ray absorption and resonant inelastic x-ray scattering, Phys. Rev. Lett. 80 (1998) 794-797. doi:10.1103/PhysRevLett.80.794.
[86] M. Rohlfing, S. G. Louie, Electron-hole excitations in semiconductors and insulators, Phys. Rev. Lett. 81 (1998) 23122315. doi:10.1103/PhysRevLett.81.2312.

[87] X. Blase, P. Boulanger, F. Bruneval, M. Fernandez-Serra, I. Duchemin, $g w$ and bethe-salpeter study of small water clusters, J. Chem. Phys. 144 (3) (2016) 034109. doi:http://dx.doi.org/10.1063/1.4940139.

[88] http://https://bse.pnl.gov/bse/portal/.

[89] D. Feller, The role of databases in support of computational chemistry calculations, J. Comp. Chem. 17 (1996) 1571. doi:10.1002/jcc.9.

[90] K. Schuchardt, B. Didier, T. Elsethagen, L. Sun, V. Gurumoorthi, J. Chase, J. Li, T. Windus, Basis set exchange: A community database for computational sciences, J. Chem. Inf. Model. 47 (2007) 1045. doi:10.1021/ci600510j.

[91] M. Valiev, E. Bylaska, N. Govind, K. Kowalski, T. Straatsma, H. V. Dam, D. Wang, J. Nieplocha, E. Apra, T. Windus, W. de Jong, Nwchem: A comprehensive and scalable open-source solution for large scale molecular simulations, Comput. Phys. Commun. 181 (9) (2010) 1477 - 1489. doi:http://dx.doi.org/10.1016/j.cpc.2010.04.018.

[92] F. Weigend, A. Köhn, C. Hättig, Efficient use of the correlation consistent basis sets in resolution of the identity mp2 calculations, J. Chem. Phys. 116 (8) (2002) 3175-3183. doi:http://dx.doi.org/10.1063/1.1445115.

[93] F. Weigend, M. Häser, H. Patzelt, R. Ahlrichs, Ri-mp2: optimized auxiliary basis sets and demonstration of efficiency, Chem. Phys. Lett. 294 (13) (1998) 143 - 152. doi:http://dx.doi.org/10.1016/S0009-2614(98)00862-8.

[94] M. Rohlfing, P. Krüger, J. Pollmann, Efficient scheme for $g w$ quasiparticle band-structure calculations with applications to bulk si and to the si(001)-(2×1) surface, Phys. Rev. B 52 (1995) 1905-1917. doi:10.1103/PhysRevB.52.1905.

[95] T. H. Dunning, Gaussian basis sets for use in correlated molecular calculations. i. the atoms boron through neon and hydrogen, J. Chem. Phys. 90 (2) (1989) 1007-1023. doi:http://dx.doi.org/10.1063/1.456153.

[96] R. A. Kendall, T. H. Dunning, R. J. Harrison, Electron affinities of the firstrow atoms revisited. systematic basis sets and wave functions, J. Chem. Phys. 96 (9) (1992) 6796-6806. doi:http://dx.doi.org/10.1063/1.462569.

[97] D. G. Truhlar, Basis-set extrapolation, Chem. Phys. Lett. $294 \quad(1-3) \quad$ (1998) $45 \quad$ - 48. doi:http://dx.doi.org/10.1016/S0009-2614(98)00866-5.

[98] F. Bruneval, X. Gonze, Accurate $G W$ self-energies in a plane-wave basis using only a few empty states: Towards large systems, Phys. Rev. B 78 (2008) 085125. doi:10.1103/PhysRevB.78.085125.

[99] J. Deslippe, G. Samsonidze, M. Jain, M. L. Cohen, S. G. Louie, Coulomb-hole summations and energies for $g w$ calculations with limited number of empty orbitals: A modified static remainder approach, Phys. Rev. B 87 (2013) 165124. doi:10.1103/PhysRevB.87.165124.

[100] M. Shao, F. H. da Jornada, C. Yang, J. Deslippe, S. G. Louie, Structure preserving parallel algorithms for solving the BetheSalpeter eigenvalue problem, Linear Algebra Appl. 488 (2016) 148-167. doi:http://dx.doi.org/10.1016/j.laa.2015.09.036.

[101] L. S. Blackford, J. Choi, A. Cleary, E. D'Azevedo, J. W. Demmel, I. Dhillon, J. J. Dongarra, S. J. Hammarling, G. Henry, A. Petitet, K. Stanley, D. W. Walker, R. C. Whaley, ScaLAPACK User's Guide, SIAM, Philadelphia, PA, USA, 1997.

[102] F. Bruneval, MoLGw: a small but accurate many-body perturbation theory code, https://github.com/bruneval/molgw (2016). 